\title{
A Highly Efficient Gold-Catalyzed Atom-Economical Annulation of Phenols with Dienes
}

\author{
Rene-Viet Nguyen, Xiaoquan Yao, and Chao-Jun Li* \\ Department of Chemistry, McGill University, 801 Sherbrooke St. West, Montreal, Quebec H3A 2K6, Canada \\ Email:cj.li@mcgill.ca
}

\section{Supporting information}

All experiments were carried out under a nitrogen atmosphere. Dichloromethane was dried using $\mathrm{CaH}_{2}$. Column chromatography was performed over Sorbent silica gel $30-60 \mu \mathrm{m} .{ }^{1} \mathrm{H}-$ NMR spectra were acquired by Varian 400 and $100 \mathrm{MHz}$ or 300 and $75 \mathrm{MHz}$, respectively, and referenced to the internal solvent signals. IR spectra were recorded by ABB Bomem MB100 spectrometer. Mass spectra data and HRMS were obtained by Kratos MS25RFA Mass Spectometer.

\section{Typical experimental procedure (Table 2, entry 1):}

A solution of $\mathrm{AuCl}_{3}$ (7.6 mg, $\left.0.025 \mathrm{mmol}\right)$ and AgOTf (19.3 mg, $\left.0.075 \mathrm{mmol}\right)$ was stirred in dry dichloromethane $(1.5 \mathrm{~mL})$ at room temperature for 2 hrs. Phenol (94 mg, $0.5 \mathrm{mmol})$ was then added, which was followed by the dropwise (approx. 1 drop/sec.) addition of cyclohexadiene $(124 \mu \mathrm{L}, 1.0 \mathrm{mmol}$ diluted in $0.5 \mathrm{~mL}$ of dry dichloromethane) and the resulting solution was stirred overnight at $40-45^{\circ} \mathrm{C}$. The resulting solution was filtered through a cotton plug and the solvent was removed under reduced pressure to afford a dark oil. The internal standard (nitromethane) was added and a crude ${ }^{1} \mathrm{H}$ NMR was recorded. The product was isolated via column chromatography on silica gel(gradiant eluent: hexane: dichloromethane $=40: 1$ to $2: 1$ ) to afford $74 \%$ of a colorless oil. 
1,2,3,4,4a,9b-Hexahydro-dibenzofuran(1a). ${ }^{1}$ The ratio of two diastereoisomers is 3:1. Syn isomer: ${ }^{1} \mathrm{H} \mathrm{NMR}\left(\mathrm{CDCl}_{3}, 400 \mathrm{MHz}\right.$, ppm): $\delta=4.68(\mathrm{q}, 1 \mathrm{H}, J=7.2 \mathrm{~Hz}), 3.20(\mathrm{q}, 1 \mathrm{H}, J=7.2 \mathrm{~Hz}) ;{ }^{13} \mathrm{C}$ $\mathrm{NMR}\left(\mathrm{CDCl}_{3}, 75 \mathrm{MHz}, \mathrm{ppm}\right): \delta=159.43,133.64,127.93,123.71$,

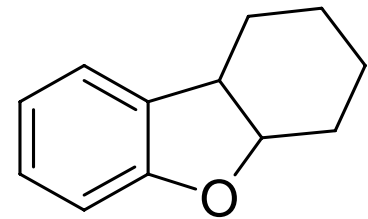
120.62, 110.22, 82.82, 41.02, 28.71, 27.99, 22.51, 21.05; Anti isomer: ${ }^{1} \mathrm{H} \mathrm{NMR}\left(\mathrm{CDCl}_{3}\right.$, 400MHz, ppm): $\delta=4.59(\mathrm{~m}, 1 \mathrm{H}), 3.00(\mathrm{~m}, 1 \mathrm{H}) ;{ }^{13} \mathrm{C} \mathrm{NMR}\left(\mathrm{CDCl}_{3}, 75 \mathrm{MHz}, \mathrm{ppm}\right): \delta=156.38$, 128.69, 127.56, 119.46, 115.35, 109.65, 71.23, 33.75, 33.58, 31.92, 29.91, 17.71; Overlapped peaks: : ${ }^{1} \mathrm{H}$ NMR(CDCl $\left.3,400 \mathrm{MHz}, \mathrm{ppm}\right): \delta=7.16-1.10(\mathrm{~m}), 6.88-6.81(\mathrm{~m}), 2.00-1.75(\mathrm{~m})$, $1.56-1.33(\mathrm{~m})$.

\section{8-Bromo-1,2,3,4,4a,9b-hexahydro-dibenzofuran(3a).}

The ratio of two diastereoisomers is 3:1. IR(neat): $\mathrm{v}_{\max }=2926$, 1600, 1446, 1414, 1369, 1217, 1085, 994 $\mathrm{cm}^{-1}$; Syn isomer: ${ }^{1} \mathrm{H}$<smiles>Brc1ccc2c(c1)C1CCCCC1O2</smiles>
$\mathrm{NMR}\left(\mathrm{CDCl}_{3}, 400 \mathrm{MHz}, \mathrm{ppm}\right): \delta=7.22(\mathrm{~s}, 1 \mathrm{H}), 7.20(\mathrm{~d}, 1 \mathrm{H}, J=8.4 \mathrm{~Hz}), 6.70(\mathrm{~d}, 1 \mathrm{H}, J=8.4 \mathrm{~Hz})$, 4.69(m, 1H), 3.20(m, 1H); $\left.{ }^{13} \mathrm{C} \mathrm{NMR(CDCl} 3,75 \mathrm{MHz}, \mathrm{ppm}\right): \delta=158.75,134.20,130.72$, 126.88, 117.24, 111.84, 83.42, 40.92, 28.32, 27.68, 22.17, 20.70; MS(EI): m/z(\%) 252( $\left.\mathrm{M}^{+}\right)$, 173; HRMS calc'd for $\mathrm{C}_{12} \mathrm{H}_{13} \mathrm{O}^{79} \mathrm{Br}$ : 252.0150; found, 252.0141; HRMS calc'd for $\mathrm{C}_{12} \mathrm{H}_{13} \mathrm{O}^{81} \mathrm{Br}$ : 254.0129; found, 254.0123; Anti isomer: ${ }^{1} \mathrm{H}$ NMR(CDCl $\left.3,400 \mathrm{MHz}, \mathrm{ppm}\right): \delta=$ 7.23(s, 1H), 7.19(d, $1 \mathrm{H}, J=8.4 \mathrm{~Hz}), 6.68(\mathrm{~d}, 1 \mathrm{H}, J=8.4 \mathrm{~Hz}), 4.58(\mathrm{~m}, 1 \mathrm{H}), 2.96(\mathrm{~m}, 1 \mathrm{H}) ;{ }^{13} \mathrm{C}$ $\mathrm{NMR}\left(\mathrm{CDCl}_{3}, 75 \mathrm{MHz}, \mathrm{ppm}\right): \delta=155.64,131.22,130.40,127.76,113.11,112.43,71.35$, 33.32, 31.68, 29.33, 28.99, 17.32; Overlapped peaks: ${ }^{1} \mathrm{H} \mathrm{NMR}\left(\mathrm{CDCl}_{3}, 400 \mathrm{MHz}, \mathrm{ppm}\right)$ : $\delta=1.98-1.78(\mathrm{~m}), 1.57-1.47(\mathrm{~m}), 1.39-1.35(\mathrm{~m})$.

\section{1,3,4-Trimethyl-5a,6,7,8,9,9a-hexahydro-dibenzofuran-2-}

$\mathbf{o l}(\mathbf{4 a}){ }^{2}$ The eluent gradient was a mixture of hexanes: ethyl acetate (60:1). The ratio of two diastereoisomers is $12: 1$. Syn isomer: ${ }^{1} \mathrm{H}$ $\operatorname{NMR}\left(\mathrm{CDCl}_{3}, 400 \mathrm{MHz}, \mathrm{ppm}\right): \delta=4.48(\mathrm{~m}, 1 \mathrm{H}), 4.24(\mathrm{~s}, 1 \mathrm{H}), 2.16(\mathrm{~s}$,

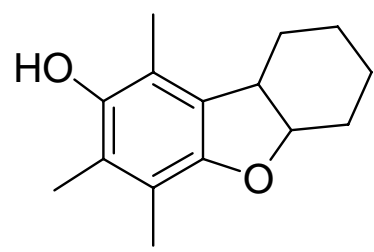
$9 \mathrm{H}) ;{ }^{13} \mathrm{C} \mathrm{NMR}\left(\mathrm{CDCl}_{3}, 100 \mathrm{MHz}, \mathrm{ppm}\right): \delta=151.51,145.90,131.33,127.14,116.77,81.86$, 40.78, 29.05, 27.96, 23.34, 20.81, 12.58, 12.57, 12.25; Anti isomer: ${ }^{1} \mathrm{H} \mathrm{NMR}_{(\mathrm{CDCl}}$, $400 \mathrm{MHz}, \mathrm{ppm}): \delta=4.82(\mathrm{~m}, 1 \mathrm{H}), 4.22(\mathrm{~s}, 1 \mathrm{H}), 3.31(\mathrm{~m}, 1 \mathrm{H}), 2.13(\mathrm{~s}, 9 \mathrm{H}) ;{ }^{13} \mathrm{C} \mathrm{NMR}\left(\mathrm{CDCl}_{3}\right.$, 100MHz, ppm): $\delta=151.32,133.82,121.83,117.35,115.70,86.10,47.75,31.84,31.65,29.64$, 
23.74, 12.48, 12.24, 11.98; Overlapped peaks: ${ }^{1} \mathrm{H} \mathrm{NMR}\left(\mathrm{CDCl}_{3}, 400 \mathrm{MHz}, \mathrm{ppm}\right)$ : $\delta=2.97(\mathrm{~m}), 2.30(\mathrm{~m}), 1.95(\mathrm{~m}), 1.76-1.56(\mathrm{~m}), 1.26-1.13(\mathrm{~m})$.

8-Methyl-1,2,3,4,4a,9b-hexahydro-dibenzofuran(2a). The ratio of two diastereoisomers is 4:1. IR(neat): 2930, 1616, 1514, 1448, 1213, 948, 813 $\mathrm{cm}^{-1}$; Syn isomer: ${ }^{1} \mathrm{H} \mathrm{NMR}\left(\mathrm{CDCl}_{3}, 400 \mathrm{MHz}, \mathrm{ppm}\right)$ :

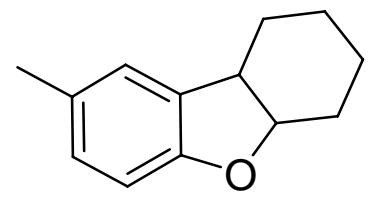
$\delta=6.95(\mathrm{~d}, 1 \mathrm{H}, J=8.0 \mathrm{~Hz}), 6.74(\mathrm{~d}, 1 \mathrm{H}, J=8.0 \mathrm{~Hz}), 4.66(\mathrm{~m}, 1 \mathrm{H}), 3.17(\mathrm{q}, 1 \mathrm{H}, J=7.2 \mathrm{~Hz}), 2.32(\mathrm{~s}$, $3 \mathrm{H}) ;{ }^{13} \mathrm{C} \mathrm{NMR}\left(\mathrm{CDCl}_{3}, 75 \mathrm{MHz}, \mathrm{ppm}\right): \delta=157.44,133.77,129.90,128.26,124.44,109.76$, 82.80, 40.97, 28.59, 27.87, 22.41, 21.14, 20.95; MS(EI): m/z(\%) 188(M+1 100$), 173$; HRMS calc'd forC ${ }_{18} \mathrm{H}_{16} \mathrm{O}$ : 188.1201; found, 188.1195; Anti isomer: ${ }^{1} \mathrm{H} \mathrm{NMR}\left(\mathrm{CDCl}_{3}, 400 \mathrm{MHz}\right.$, ppm): $\delta=6.95(\mathrm{~d}, 1 \mathrm{H}, J=8.0 \mathrm{~Hz}), 6.75(\mathrm{~d}, 1 \mathrm{H}, J=8.0 \mathrm{~Hz}), 4.59(\mathrm{~m}, 1 \mathrm{H}), 2.97(\mathrm{~m}, 1 \mathrm{H}), 229(\mathrm{~s}$, $3 \mathrm{H}) ;{ }^{13} \mathrm{C} \mathrm{NMR}\left(\mathrm{CDCl}_{3}, 75 \mathrm{MHz}, \mathrm{ppm}\right): \delta=154.29,129.18,128.52,128.23,126.46,115.07$, 70.99, 33.65, 33.38, 31.75, 29.84, 20.73, 17.58; Overlapped peaks: ${ }^{1} \mathrm{H} \mathrm{NMR}\left(\mathrm{CDCl}_{3}\right.$, 400MHz, ppm): 6.98(s), 1.98(m), 1.90-1.54, 1.57-1.53(m), 1.37(m).

\section{6b,7,8,9,10,10a-Hexahydro-benzo[b]naphtho[2,1-d]furan(5a). ${ }^{4}$ The} ratio of two diastereoisomers is $11: 1$. Syn isomer: ${ }^{1} \mathrm{H}-\mathrm{NMR}\left(\mathrm{CDCl}_{3}\right.$, 400MHz, ppm): $\delta=7.88(\mathrm{~d}, 1 \mathrm{H}, J=8.4 \mathrm{~Hz}), 7.74(\mathrm{~d}, 1 \mathrm{H}, J=6.4 \mathrm{~Hz})$, 7.52(t, $1 \mathrm{H}, J=7.2 \mathrm{~Hz}), 7.35(\mathrm{t}, 1 \mathrm{H}, J=7.2 \mathrm{~Hz}), 7.25(\mathrm{~d}, 1 \mathrm{H}, J=8.4 \mathrm{~Hz})$, 4.83(m, 1H), 3.48(m, $\left.1 \mathrm{H}) ;{ }^{13} \mathrm{C} \mathrm{NMR(CDCl} 3,75 \mathrm{MHz}, \mathrm{ppm}\right): \delta=156.81$,

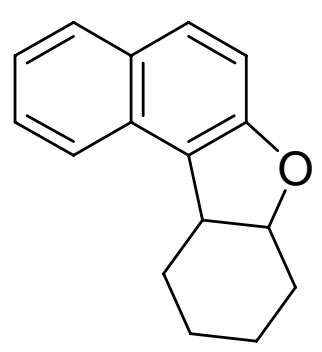
130.49, 129.67, 129.06, 128.70, 126.94, 126.61, 122.89, 122.88, 112.81, 83.81, 40.21, 29.48, 27.84, 23.03, 20.86; Anti isomer (some peaks are difficult to distinguished): ${ }^{1} \mathrm{H}-\mathrm{NMR}\left(\mathrm{CDCl}_{3}\right.$, 400MHz, ppm): $\delta=7.98(\mathrm{~d}, 1 \mathrm{H}, J=8.4 \mathrm{~Hz}), 7.59(\mathrm{t}, 1 \mathrm{H}, J=7.2 \mathrm{~Hz}), 7.18(\mathrm{~d}, 1 \mathrm{H}, J=8.4 \mathrm{~Hz})$, 4.68(m, 1H), 3.69(m, 1H); $\left.{ }^{13} \mathrm{C} \mathrm{NMR(CDCl} 3,75 \mathrm{MHz}, \mathrm{ppm}\right): \delta=153.77,128.93,128.80$, 127.83, 126.41, 125.89, 124.03, 123.86, 123.72, 112.53, 70.96, 40.28, 27.44, 26.99, 23.56, 23.14. Overlapped peaks: ${ }^{1} \mathrm{H}-\mathrm{NMR}\left(\mathrm{CDCl}_{3}, 400 \mathrm{MHz}, \mathrm{ppm}\right): \delta=2.46(\mathrm{~m}), 2.26(\mathrm{~m}), 1.91(\mathrm{~m})$, 1.73(m), 1.32(m).

\section{7,8,9,10,11,11a-Hexahydro-6bH-12-oxa-naphtho[2,1-a]azulene(6a). ${ }^{4}$}

The ratio of two diastereoisomers is 8:1. Syn isomer: ${ }^{1} \mathrm{H}$ NMR( $\mathrm{CDCl}_{3}$, 400MHz, ppm): $\delta=7.87(\mathrm{~d}, 1 \mathrm{H}, J=8.0 \mathrm{~Hz}), 7.75(\mathrm{~d}, 1 \mathrm{H}, J=8.0 \mathrm{~Hz}), 7.71(\mathrm{~d}$, $1 \mathrm{H}, J=8.4 \mathrm{~Hz}$ ), 7.53(t, $1 \mathrm{H}, J=8.4 \mathrm{~Hz}), 7.35(\mathrm{t}, 1 \mathrm{H}, J=8.4 \mathrm{~Hz}), 7.08(\mathrm{~d}, 1 \mathrm{H}$, $J=8.4 \mathrm{~Hz}), 5.07(\mathrm{~m}, 1 \mathrm{H}), 3.82(\mathrm{~m}, 1 \mathrm{H}) ;{ }^{13} \mathrm{C} \mathrm{NMR}\left(\mathrm{CDCl}_{3}, 75 \mathrm{MHz}, \mathrm{ppm}\right)$ :

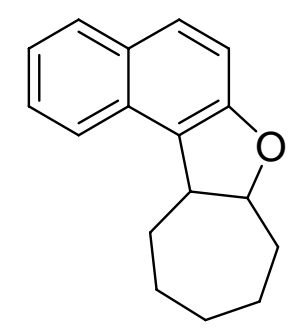


$\delta=156.58,130.83,129.77,129.52,129.37,126.73,123.14,122.73,122.49,112.26$, 88.03, 47.18, 31.99, 31.84, 29.97, 29.58, 24.22; Anti isomer (some peaks are difficult to distinguished): ${ }^{1} \mathrm{H} \mathrm{NMR}\left(\mathrm{CDCl}_{3}, 400 \mathrm{MHz}, \mathrm{ppm}\right): \delta=7.97(\mathrm{~d}, 1 \mathrm{H}, J=8.0 \mathrm{~Hz}), 7.69(\mathrm{~d}, 1 \mathrm{H}, J=8.4$ $\mathrm{Hz}), 7.62(\mathrm{~d}, 1 \mathrm{H}, J=8.0 \mathrm{~Hz}), 4.83(\mathrm{~m}, 1 \mathrm{H}), 3.75(\mathrm{~m}, 1 \mathrm{H}) ;{ }^{13} \mathrm{C} \mathrm{NMR}\left(\mathrm{CDCl}_{3}, 75 \mathrm{MHz}, \mathrm{ppm}\right)$ : $\delta=155.94,128.52,125.90,125.60,124.32$, 123.91, 123.38, 122.86, 122.35, 112.45, 73.12, 37.53, 34.82, 29.14, 28.11, 26.30, 26.24. Overlapped peaks: ${ }^{1} \mathrm{H} \mathrm{NMR}\left(\mathrm{CDCl}_{3}, 400 \mathrm{MHz}, \mathrm{ppm}\right)$ : $\delta=2.37(\mathrm{~m}), 2.19-1.97(\mathrm{~m}), 1.97(\mathrm{~m}), 1.83(\mathrm{~m}), 1.55(\mathrm{~m})$.

\section{7a,8,9,10,11,12,13,13a-Octahydro-7-oxaoctaleno[2,-a]-}

naphthalene(7a). ${ }^{4}$ The ratio of two diastereoisomers is 5:1. Syn isomer: ${ }^{1} \mathrm{H} \mathrm{NMR}\left(\mathrm{CDCl}_{3}, 300 \mathrm{MHz}, \mathrm{ppm}\right): \delta=7.85(\mathrm{~d}, 1 \mathrm{H}, J=9.6 \mathrm{~Hz})$, 7.13(d, 1H, J=9.0 Hz), 4.82(m, $1 \mathrm{H}), 3.61(\mathrm{~m}, 1 \mathrm{H}), 2.38(\mathrm{~m}, 2 \mathrm{H}) ;{ }^{13} \mathrm{C}$ $\mathrm{NMR}\left(\mathrm{CDCl}_{3}, 75 \mathrm{MHz}, \mathrm{ppm}\right): \delta=155.47,130.47,129.94,129.40$,

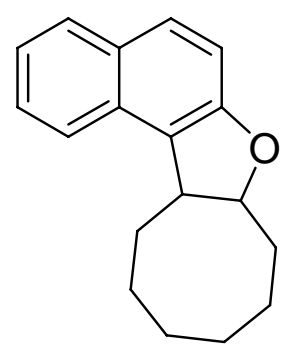
129.22, 126.87, 125.85, 122.82, 122.44, 112.21, 88.86, 45.90, 30.91, 28.59, 26.66, 26.17, 25.86, 25.67; Anti isomer (some peaks are difficult to distinguished): ${ }^{1} \mathrm{H} \mathrm{NMR}\left(\mathrm{CDCl}_{3}\right.$, 300MHz, ppm): $\delta=7.90(\mathrm{~d}, 1 \mathrm{H}, J=9.6 \mathrm{~Hz}), 7.11(\mathrm{~d}, 1 \mathrm{H}, J=9.0 \mathrm{~Hz}), 4.56(\mathrm{~m}, 1 \mathrm{H}), 3.87(\mathrm{~m}, 1 \mathrm{H})$; ${ }^{13} \mathrm{C} \mathrm{NMR}\left(\mathrm{CDCl}_{3}, 75 \mathrm{MHz}, \mathrm{ppm}\right): \delta=155.09,129.14,128.81,128.04,126.29,124.38,122.96$, 122.86, 120.09, 111.94, 73.53, 34.92, 32.28, 31.59, 29.62, 27.26, 24.51, 23.03; Overlapped peaks: ${ }^{1} \mathrm{H}$ NMR(CDCl $\left.3,300 \mathrm{MHz}, \mathrm{ppm}\right): \delta=7.67(\mathrm{~m}), 7.51(\mathrm{~m}), 7.33(\mathrm{~m}), 2.30-2.22(\mathrm{~m}), 1.91-$ 1.85(m), 1.69-1.23(m).

\section{3-Bromo-7a,8,9,10,11,11a-hexahydro-benzo[b]naphtho[1,2-}

d]furan(8a). The ratio of two diastereoisomers is $11: 1$. $\mathrm{IR}(\mathrm{KBr}$ plate): 2930, 1626, 1575, 1508, 1474, 1348, 1161, 1061 $\mathrm{cm}^{-1}$; Syn isomer: ${ }^{1} \mathrm{H}-\mathrm{NMR}\left(\mathrm{CDCl}_{3}, 300 \mathrm{MHz}, \mathrm{ppm}\right): \delta=4.79(\mathrm{~m}, 1 \mathrm{H}), 3.44(\mathrm{~m}$,

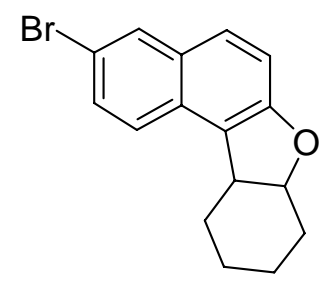
$1 \mathrm{H}) ;{ }^{13} \mathrm{C} \mathrm{NMR}\left(\mathrm{CDCl}_{3}, 75 \mathrm{MHz}, \mathrm{ppm}\right): \delta=157.27,131.01,130.76,129.89,127.95,127.89$, 127.33, 124.66, 116.39, 113.85, 83.84, 39.92, 29.30, 27.53, 22.72, 20.52; MS(EI) m/z(\%): 302( $\left.\mathrm{M}^{+}, 100\right)$, 223(100); HRMS calc'd for $\mathrm{C}_{16} \mathrm{H}_{15} \mathrm{O}^{79} \mathrm{Br}$ : 302.0306; found: 302.0297; ); HRMS calc'd for $\mathrm{C}_{16} \mathrm{H}_{15} \mathrm{O}^{81} \mathrm{Br}$ : 304.0286; found: 304.0282; Anti isomer: ${ }^{1} \mathrm{H} \mathrm{NMR}\left(\mathrm{CDCl}_{3}\right.$, 400MHz, ppm): 4.67(m, 1H), 3.62(m, $\left.1 \mathrm{H}) ;{ }^{13} \mathrm{C} \mathrm{NMR(CDCl} 3,75 \mathrm{MHz}, \mathrm{ppm}\right): \delta=156.67$, 131.29, 130.77, 129.76, 129.10, 128.72, 126.94, 126.65, 122.92, 112.81, 71.84, 34.13, 30.52, 
27.05, 23.28, 21.85; Overlapped peaks: ${ }^{1} \mathrm{H}-\mathrm{NMR}\left(\mathrm{CDCl}_{3}, 300 \mathrm{MHz}, \mathrm{ppm}\right): \delta=7.96(\mathrm{~s})$, 7.58-4.49(m), 7.17(m), 2.38(m), 2.15(m), 1.86(m), 1.70(m), 1.55(m), 1.34-1.18(m).

\section{3-Methoxy-7a,8,9,10,11,11a-hexahydro-benzo[b]naphtho[1,2-}

d]furan(9a). The ratio of two diastereoisomers is $11: 1$. $\mathrm{IR}(\mathrm{KBr}$ plate): 2935, 2853, 1626, 1600, 1521, 1347, 1261, 1098, 733 $\mathrm{cm}^{-1}$;

Syn isomer: ${ }^{1} \mathrm{H} \mathrm{NMR}\left(\mathrm{CDCl}_{3}, 400 \mathrm{MHz}, \mathrm{ppm}\right): \delta=4.76(\mathrm{~m}, 1 \mathrm{H})$, 3.42(m, 1H); $\left.{ }^{13} \mathrm{C} \mathrm{NMR(CDCl} 3,75 \mathrm{MHz}, \mathrm{ppm}\right): \delta=155.62,155.31$,

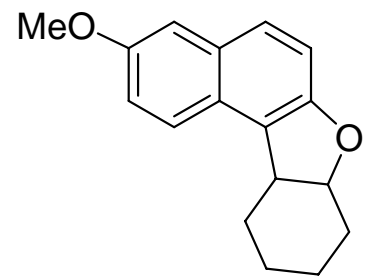
130.58, 127.50, 126.06, 124.51, 119.63, 113.11, 107.31, 83.64, 55.67, 40.34, 29.66, 27.88, 23.12, 20.92; MS(EI): m/z(\%) 254(M+100), 174; HRMS calc'd for $\mathrm{C}_{17} \mathrm{H}_{18} \mathrm{O}_{2:}$ 254.1307; found, 254.1304; Anti isomer: ${ }^{1} \mathrm{H} \mathrm{NMR}\left(\mathrm{CDCl}_{3}, 400 \mathrm{MHz}, \mathrm{ppm}\right)$ : 4.65(m, $\left.1 \mathrm{H}\right)$, 3.64(m, 1H); ${ }^{13} \mathrm{C} \mathrm{NMR}\left(\mathrm{CDCl}_{3}, 75 \mathrm{MHz}, \mathrm{ppm}\right): \delta=153.88,152.26,129.41,126.14,125.76,122.60,118.08$, 114.12, 112.10, 107.85, 70,96, 55.02, 34.26, 31.78, 24.14, 21.98, 18.43; Overlapped peaks: 7.61(m), 7.06(m), 3.91(s, 3H), 2.35(m), 2.04(m), 1.89-1.79(m), 1.71(m), 1.34-1.27(m).

\section{7a,8,9,10,11,11a-Hexahydro-benzo[b]naphtho[1,2-d]furan-6-ol(10a).}

The eluent was a mixture of hexanes:ethyl acetate (60:1). The diastereoisomers can be separated with an eluent of hexanes:dichloromethane $(1: 1)$ on a preparative TLC. One of the

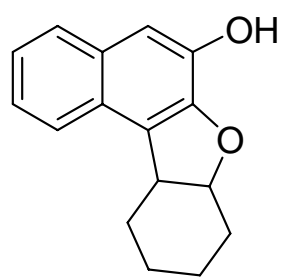
diastereoisomer: IR(KBr plate): 3409, 2932, 1629, 1593, 1525, 1458, 1381, 1299, 908, 733cm ${ }^{-1} ;{ }^{1} \mathrm{H} \mathrm{NMR}\left(\mathrm{CDCl}_{3}, 400 \mathrm{MHz}, \mathrm{ppm}\right): \delta=7.68-7.316(\mathrm{~m}, 2 \mathrm{H}), 7.29(\mathrm{~m}, 3 \mathrm{H}), 4.85(\mathrm{~m}, 1 \mathrm{H})$, 3.44(m, 1H), 2.47(m, 1H), 2.21(m, 1H), 1.86-1.60(m, 3H), $1.33-1.22(\mathrm{~m}, 2 \mathrm{H}) ;{ }^{13} \mathrm{C}$ NMR(CDCl 3 , 75MHz, ppm): 144.64, 143.70, 129.43, 127.47, 126.91, 123.87, 123.65, 121.43, 118.09, 107.81, 71.85, 33.99, 30.80, 29.76, 26.72, 18.13; MS(EI): m/z(\%) 240 ( $\left.\mathrm{M}^{+}, 100\right), 223$, 160; HRMS calc'd for $\mathrm{C}_{16} \mathrm{H}_{16} \mathrm{O}_{2}$ : 240.1153; found: 240.1147. Another diastereoisomer: ${ }^{1} \mathrm{H}$ $\mathrm{NMR}\left(\mathrm{CDCl}_{3}, 400 \mathrm{MHz}, \mathrm{ppm}\right): \delta=7.72(\mathrm{~m}, 1 \mathrm{H}), 7.70(\mathrm{~m}, 1 \mathrm{H}), 7.34(\mathrm{~m}, 2 \mathrm{H}), 7.18(\mathrm{~m}, 1 \mathrm{H})$, 5.59(s, 1H), 4.87(m, 1H), 3.50(m, 1H), 2.40(m, 1H), 2.21(m, 1H), 1.88(m, 1H), 1.72-1.61(m, 3H), 1.33-1.28(m, 2H); $\left.{ }^{13} \mathrm{C} \mathrm{NMR(CDCl} 3,75 \mathrm{MHz}, \mathrm{ppm}\right):$ 146.87, 142.02, 131.04, 128.19, 127.77, 125.73, 124.25, 123.72, 122.82, 110.39, 85.25, 40.79, 29.18, 27.60, 27.72, 20.57. 


\section{1,2,3,4,4a,8c,9,10,11,12,12a,14a-Dodecahydro-13,14-dioxa-}

benzo[c]indeno[2,1-a]fluorene(10b). Two isomers can be separated with an eluent of hexanes:dichloromethane (1:1) on a preparative TLC. One of the isomer: $\operatorname{IR}(\mathrm{KBr}$ plate): 2929, 1599, 1527, 1456, 1370, 1293, 1178, 1113, $1036 \mathrm{~cm}^{-1} .{ }^{1} \mathrm{H}$ NMR(CDCl $\left.3,400 \mathrm{MHz}, \mathrm{ppm}\right): \delta=7.68-(\mathrm{m}, 2 \mathrm{H})$, 7.29(m, 2H), 4.85(m, 2H), 3.44(m, 2H), 2.47(m, 2H), 2.21(m, 2H), 1.86(m,

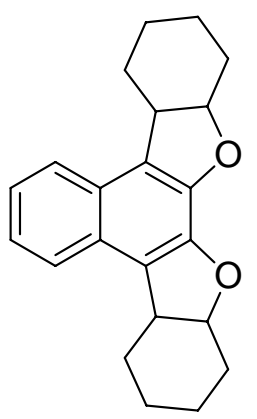

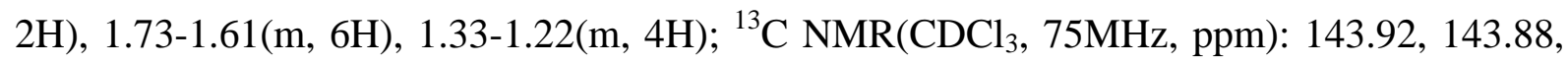
128.77, 128.71, 126.23, 126.20, 123.88, 123.87, 123.58, 123.57, 85.42, 85.00, 40.43, 40.42, 29.63, 29.55, 27.67, 27.61, 23.06, 23.02, 20.74, 20.73; MS(EI): m/z(\%) 320(M+, 100); HRMS calc'd for $\mathrm{C}_{22} \mathrm{H}_{24} \mathrm{O}_{2}$ : 320.1776; found: 320.1772. Another isomer: ${ }^{1} \mathrm{H} \mathrm{NMR}\left(\mathrm{CDCl}_{3}, 400 \mathrm{MHz}\right.$, ppm): $\delta=7.77(\mathrm{~m}, 2 \mathrm{H}), 7.38(\mathrm{~m}, 2 \mathrm{H}), 4.94(\mathrm{~m}, 2 \mathrm{H}), 3.53(\mathrm{~m}, 2 \mathrm{H}), 2.47(\mathrm{~m}, 1 \mathrm{H}), 2.21(\mathrm{~m}, 2 \mathrm{H})$, 1.97(m, 2H), 1.68-1.55(m, 2H), 1.35-1.20(m, 2H); $\left.{ }^{13} \mathrm{C} \mathrm{NMR(CDCl} 3,75 \mathrm{MHz}, \mathrm{ppm}\right): 153.61$, 127.91, 124.55, 123.65, 122.79, 85.54, 40.51, 29.49, 27.64, 23.30, 20.62.

\section{$\underline{\text { References }}$}

${ }^{1}$ Kurono, N.; Honda, E.; Komatsu, F.; Orito, K.; Tokuda, M. Tetrahedron 2004, 60, 1791.

${ }^{2}$ Novak, L.; Kovacs, P.; Kolonits, P.; Hanania, M.; Fekete, J.; Szabo, E.; Szantay, C. Synthesis 1997, 909.

${ }^{3}$ Novak, L.; Kovacs, P.; Pirok, G.; Kolonits, P.; Hanania, M.; Donath, K.; Szantay, C. Tetrahedron 1997, 53, 9789.

${ }^{4}$ Kovacs, P.; Kolonits, P.; Kaleta, Z.; Parkanyi, L.; Szabo, E.; Novak, L. Synthesis 2003, 1043. 


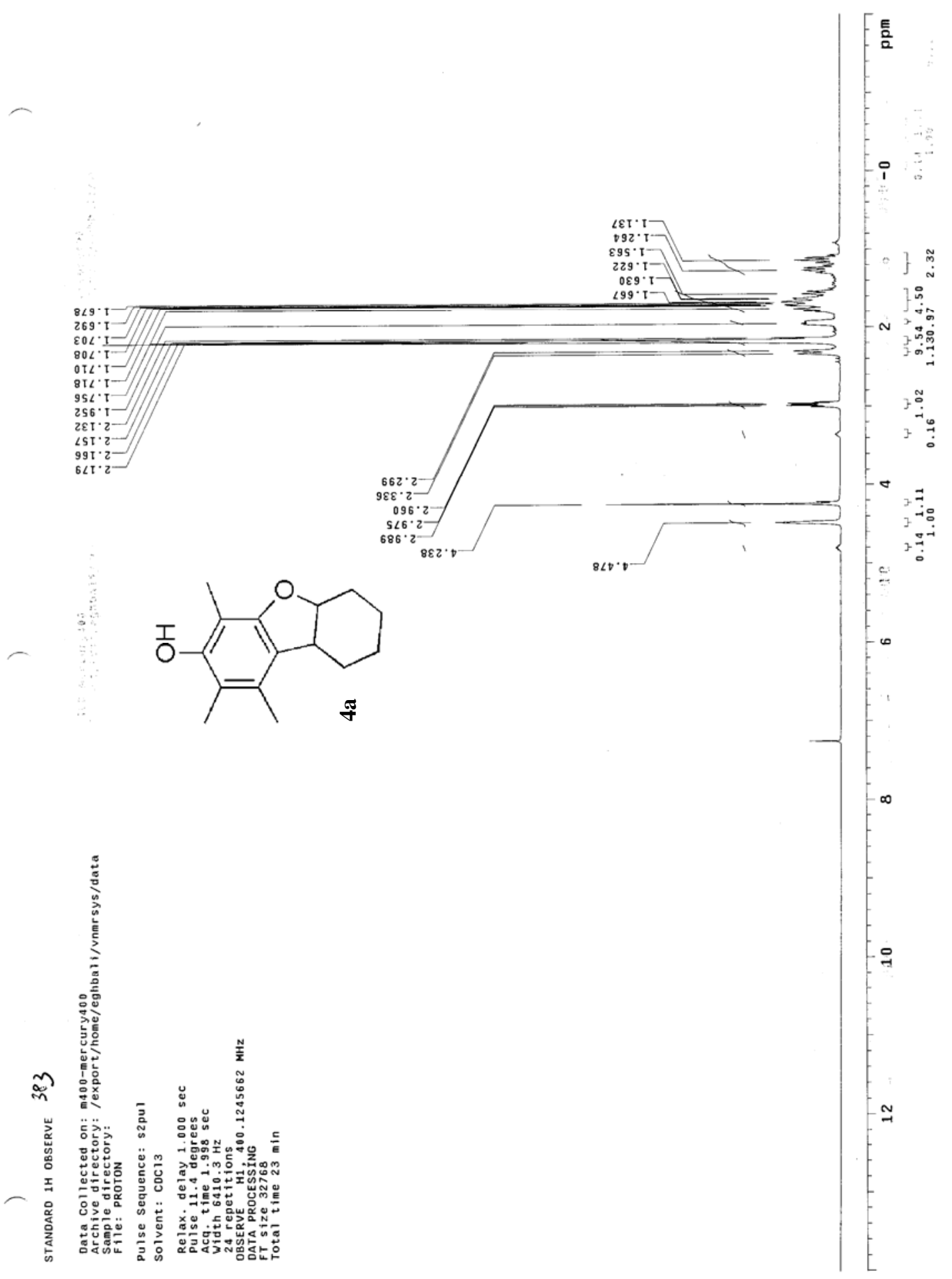




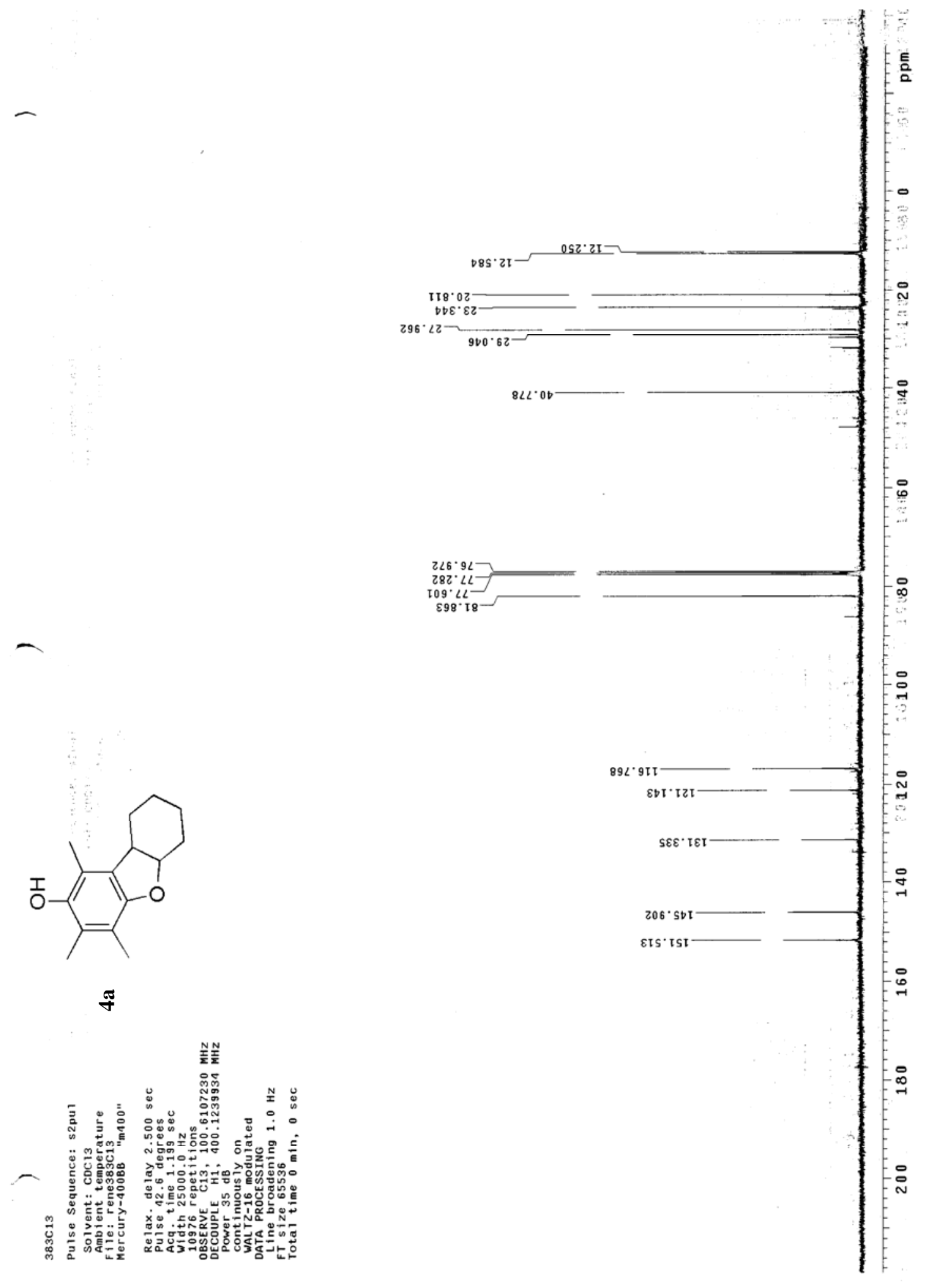



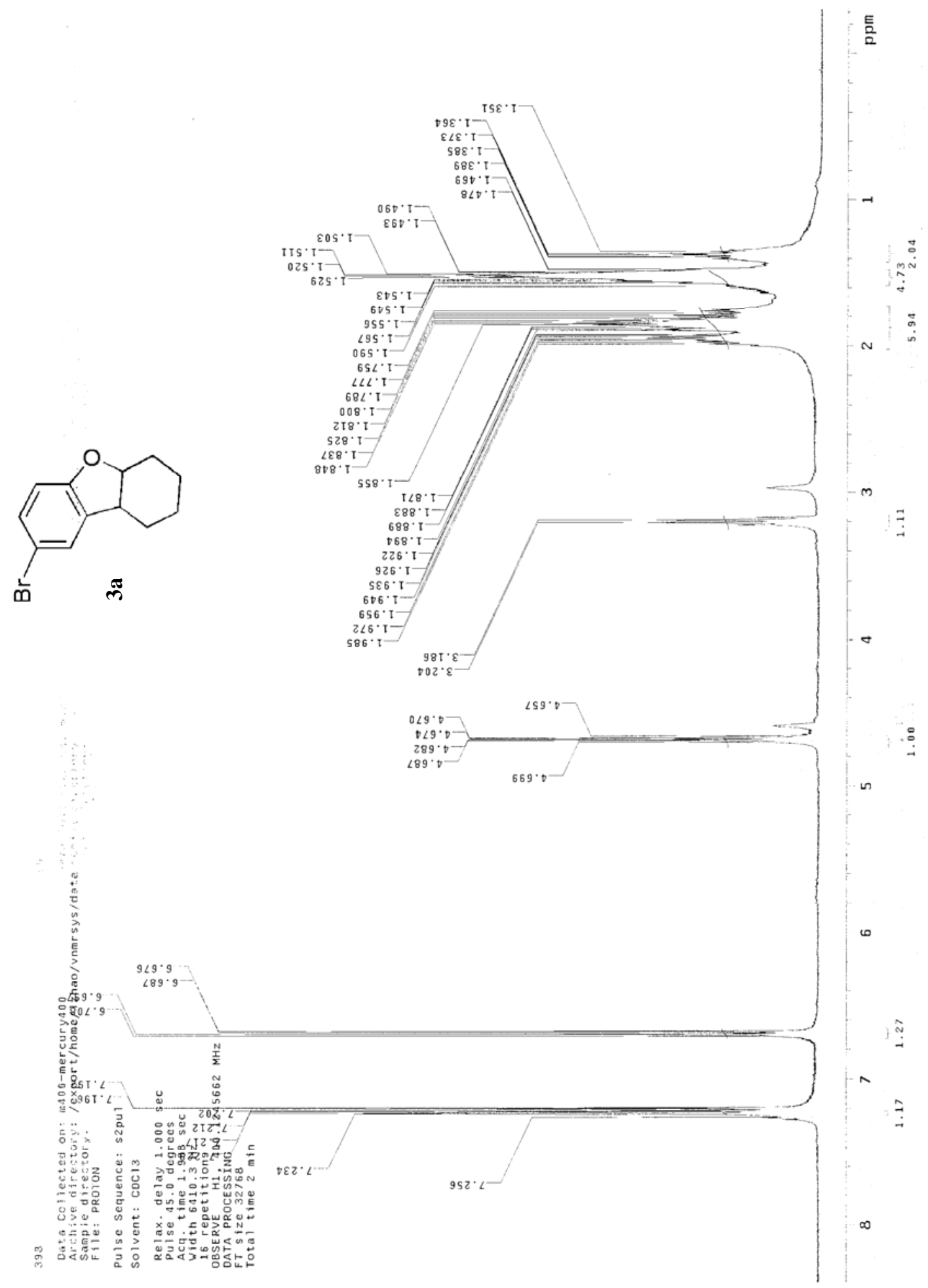


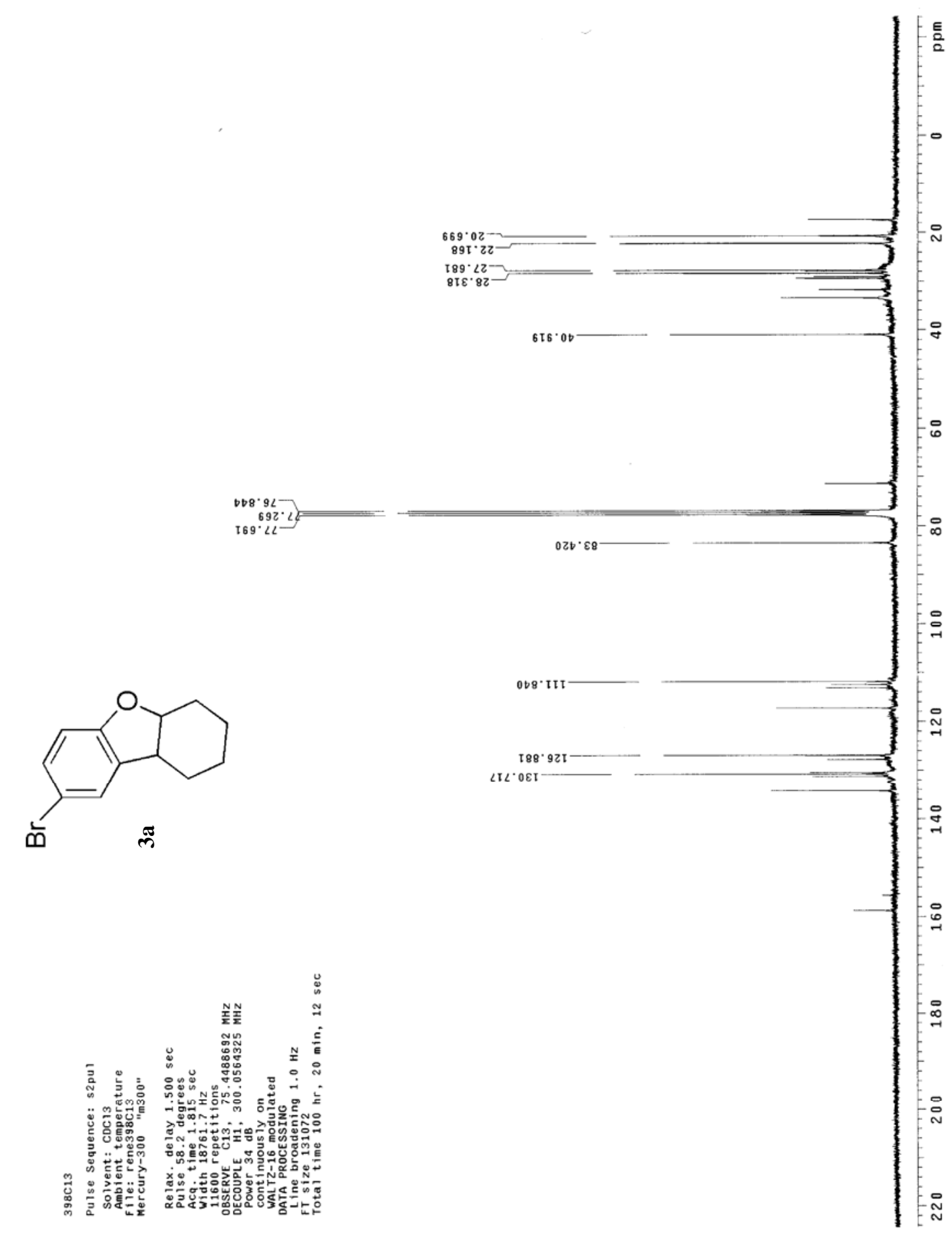




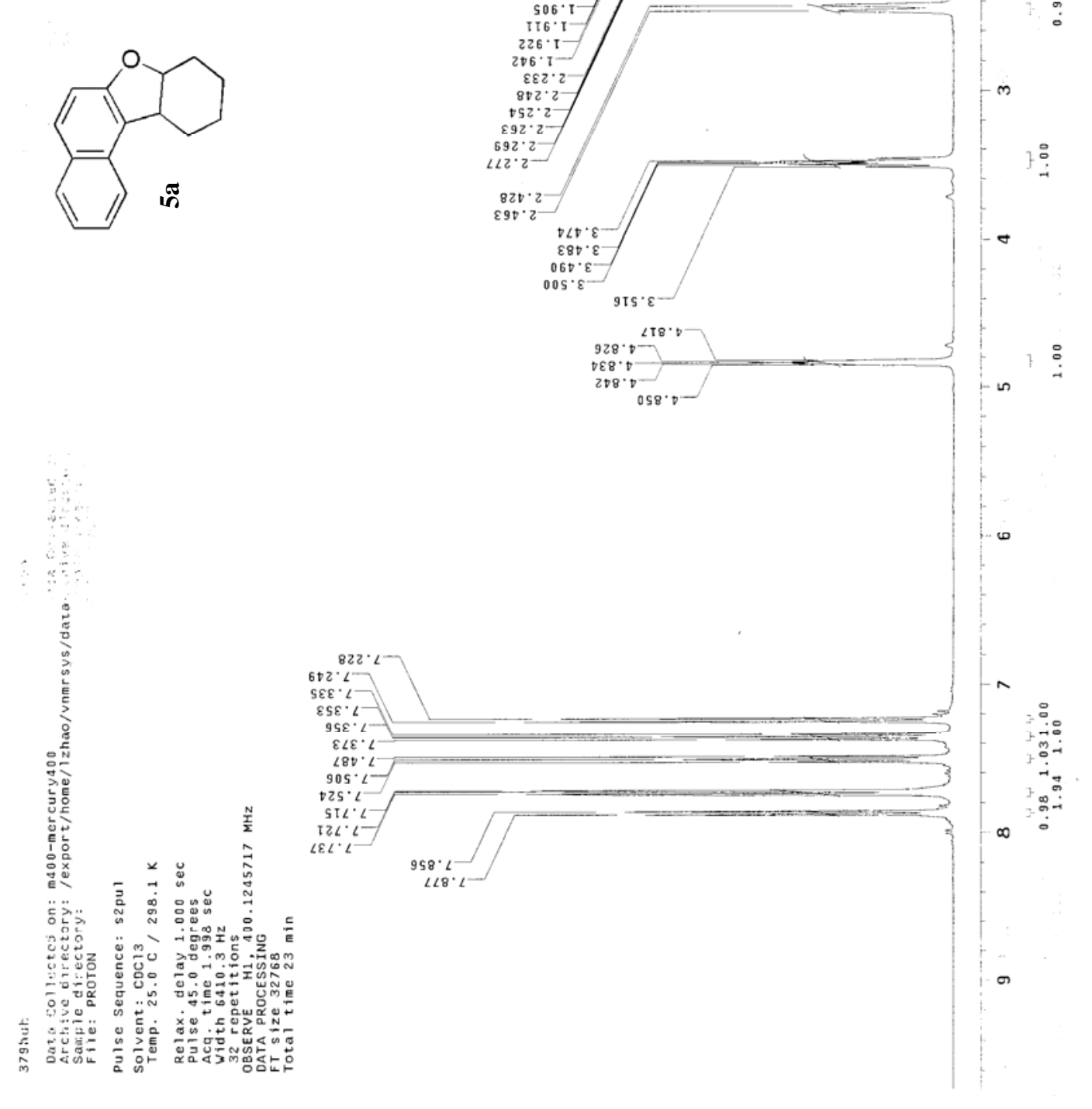




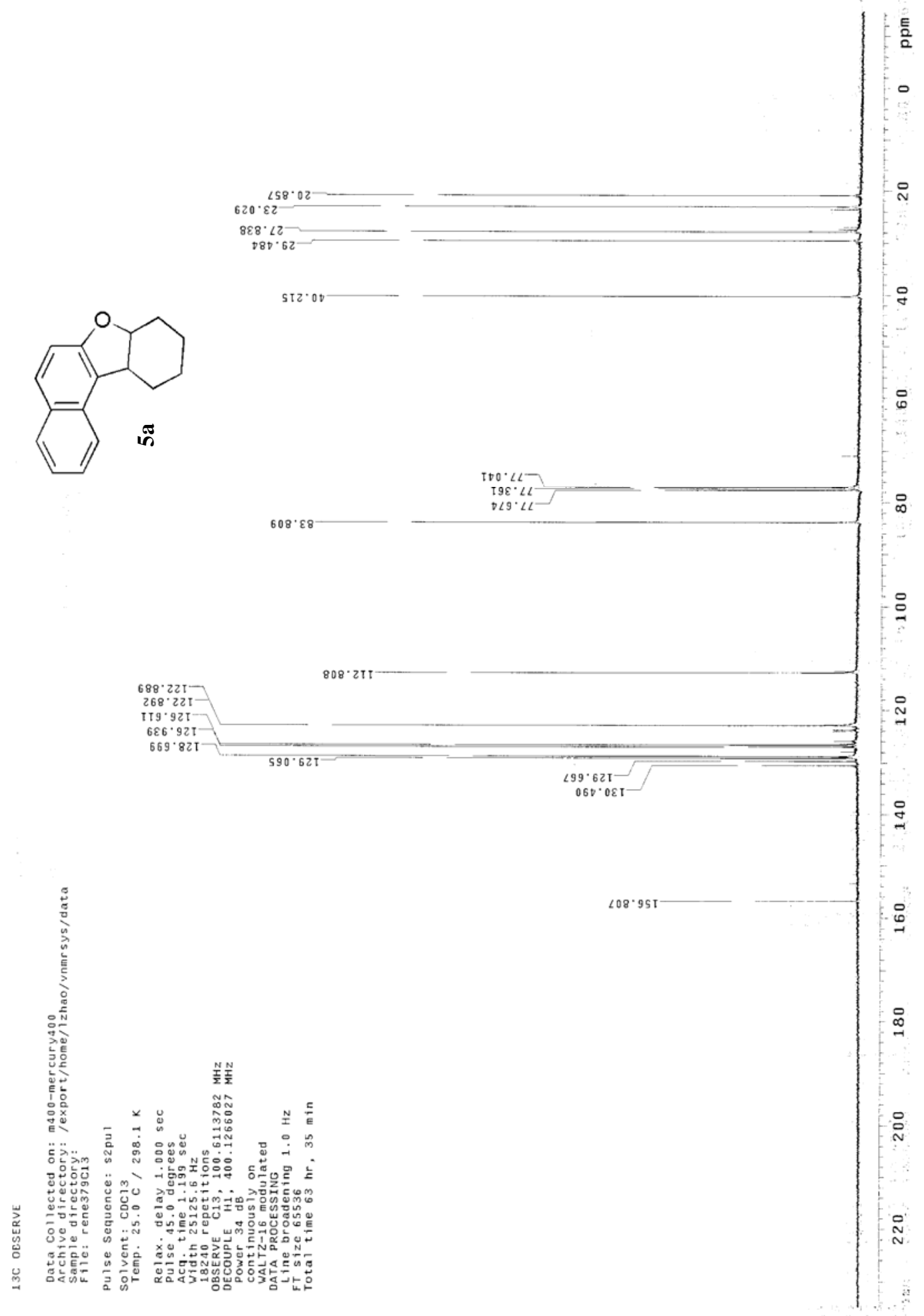



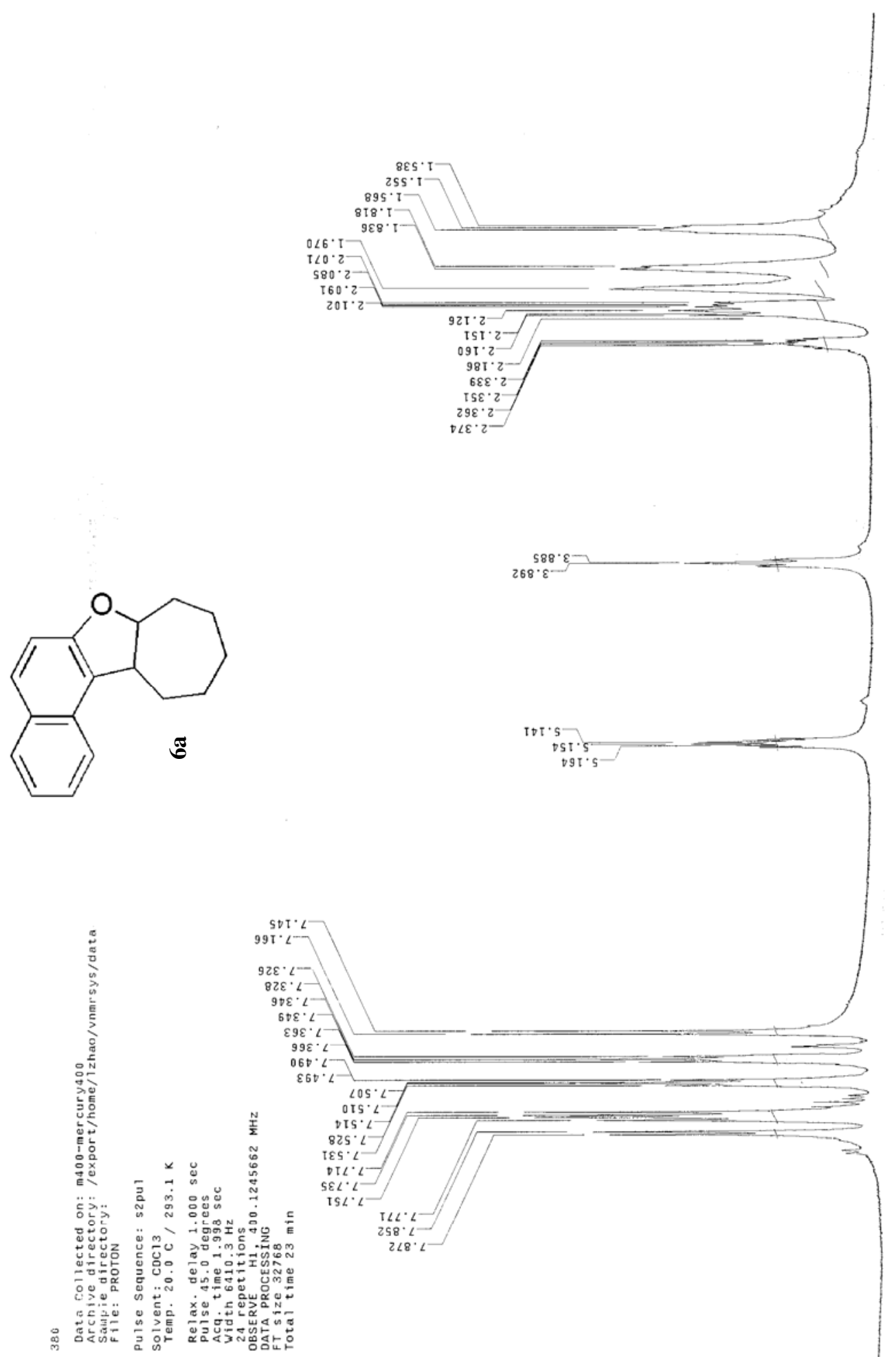


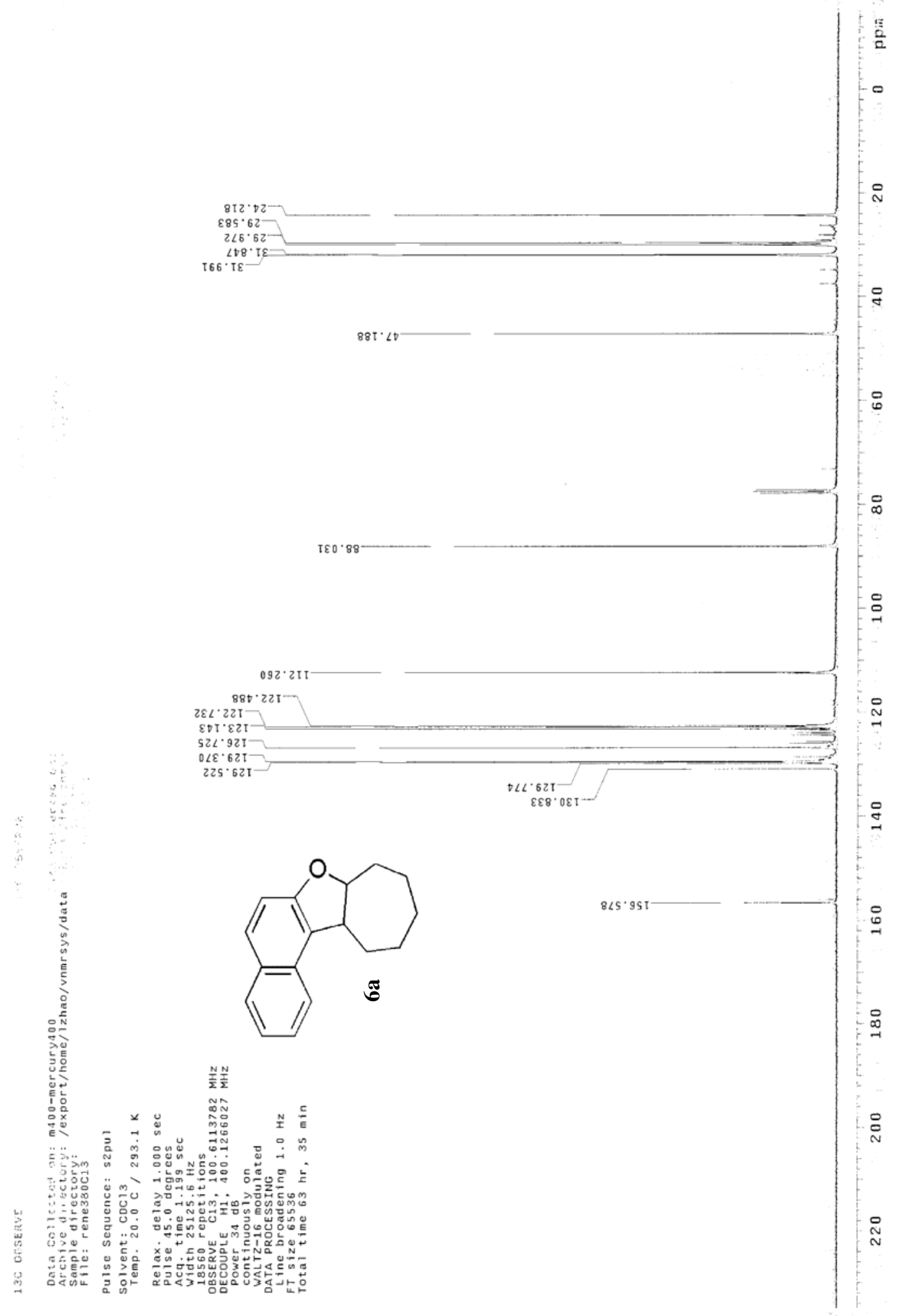



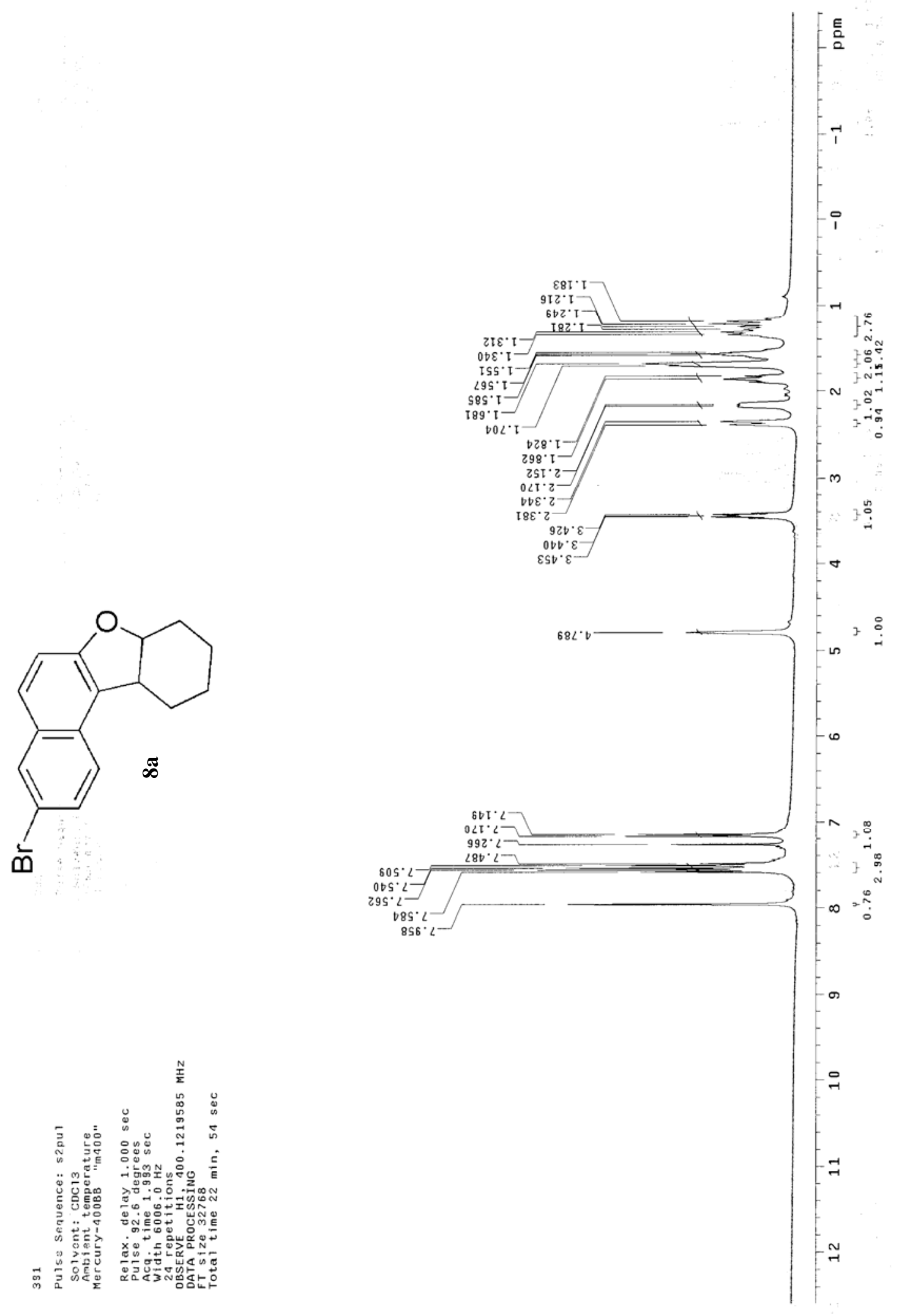


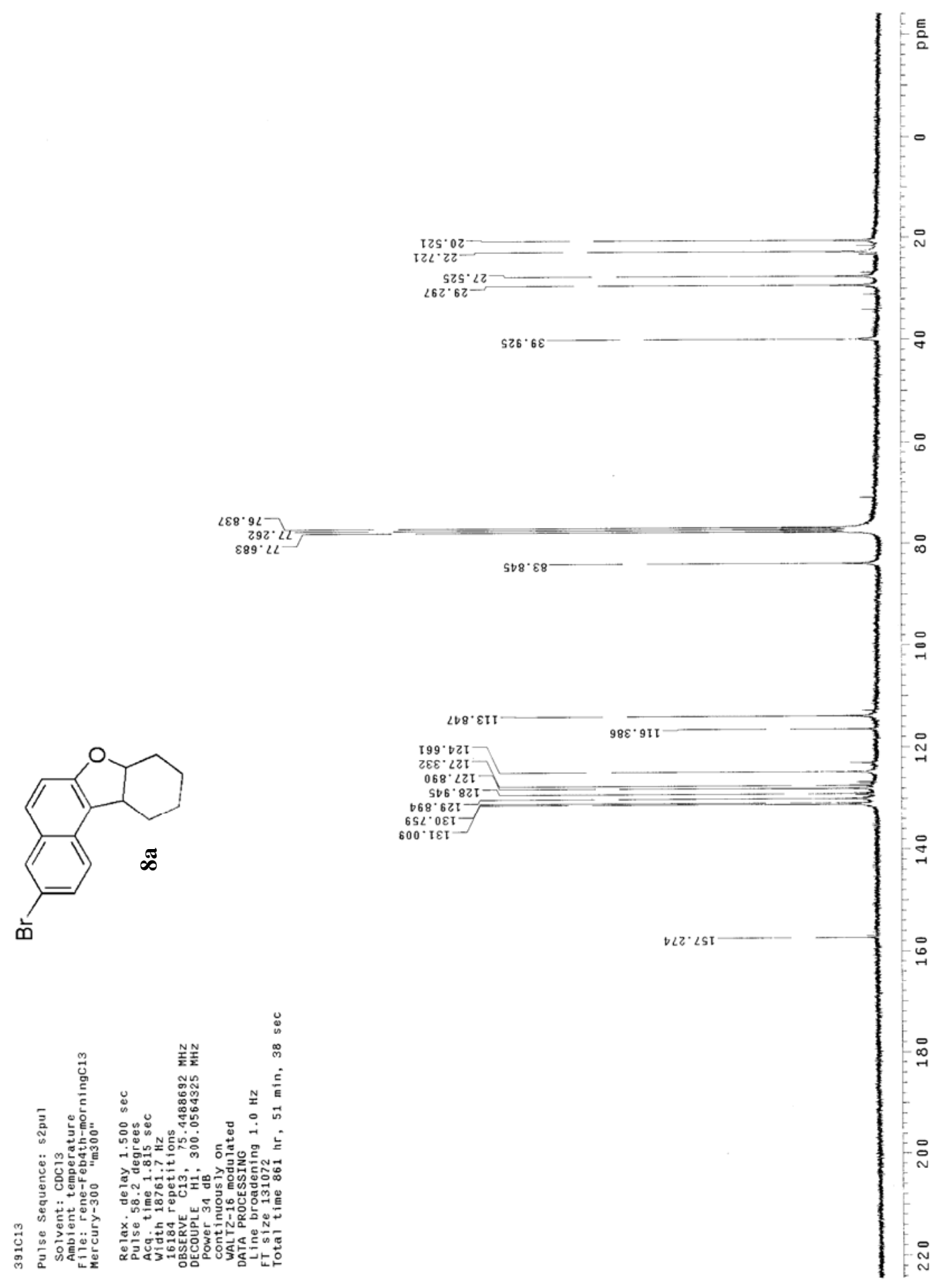




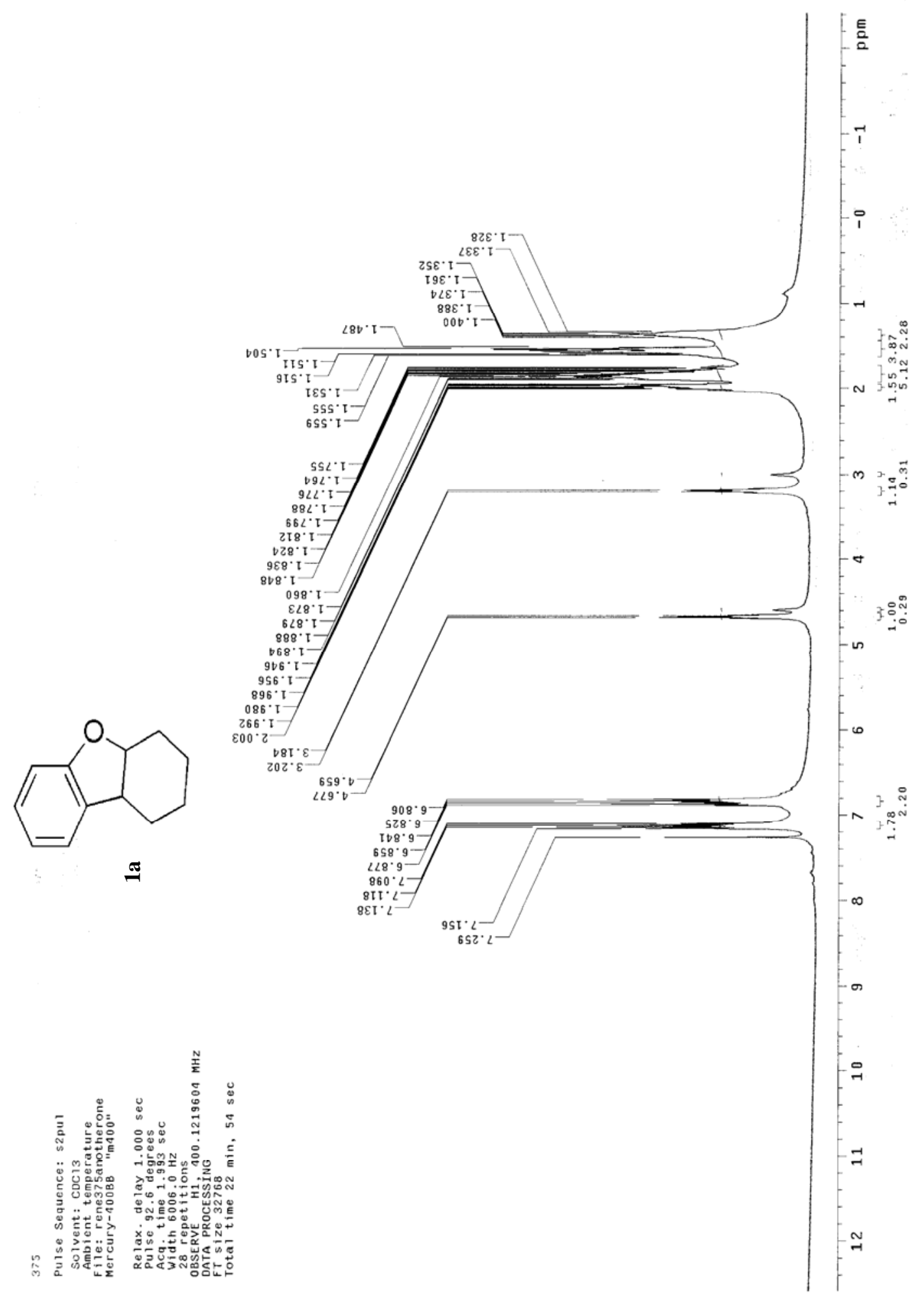



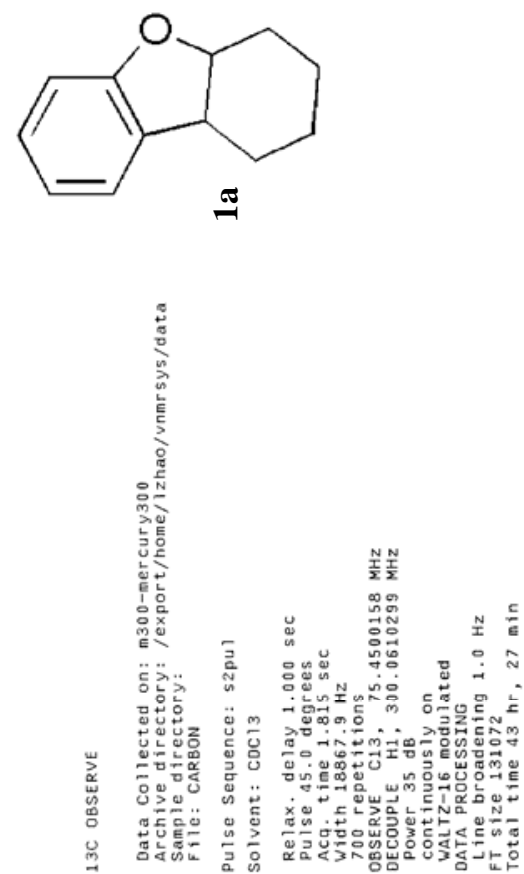

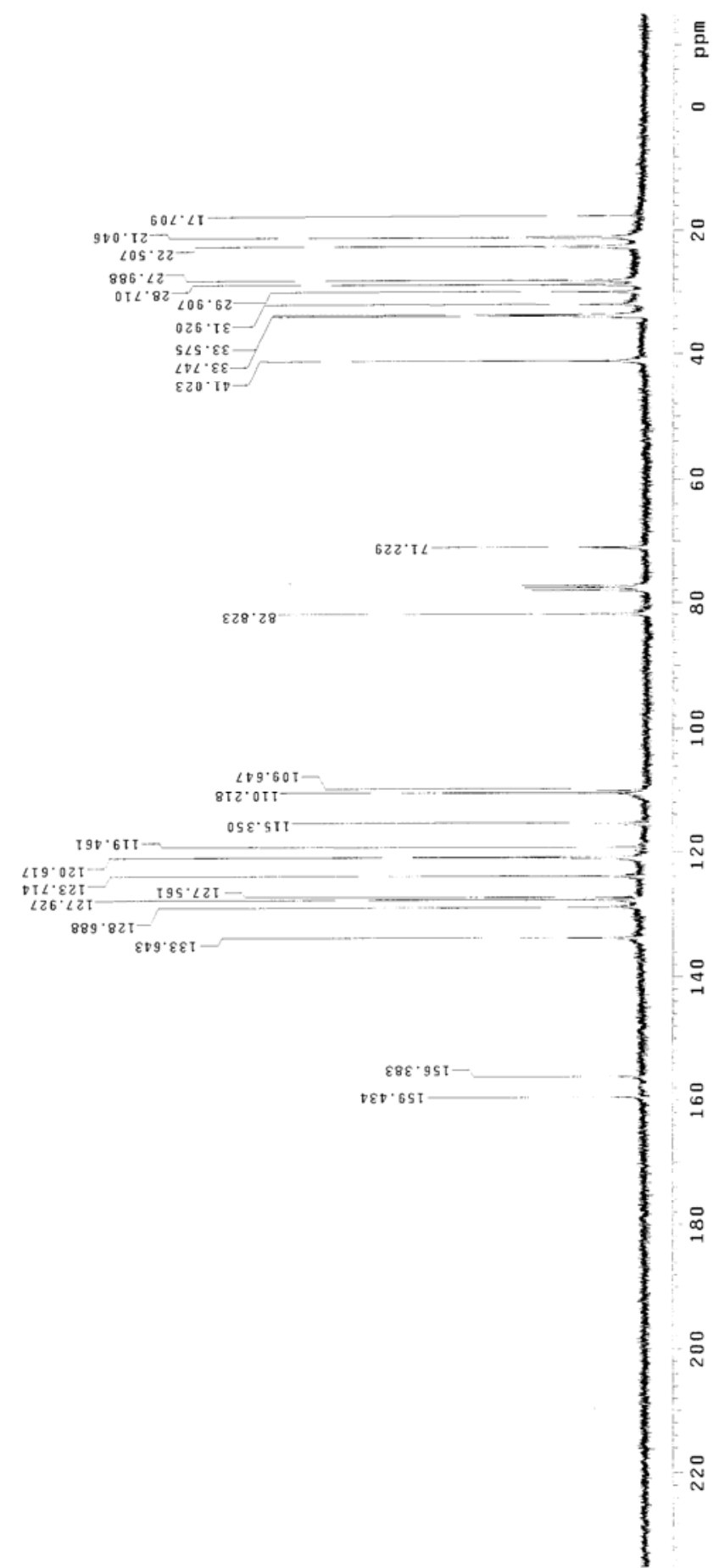




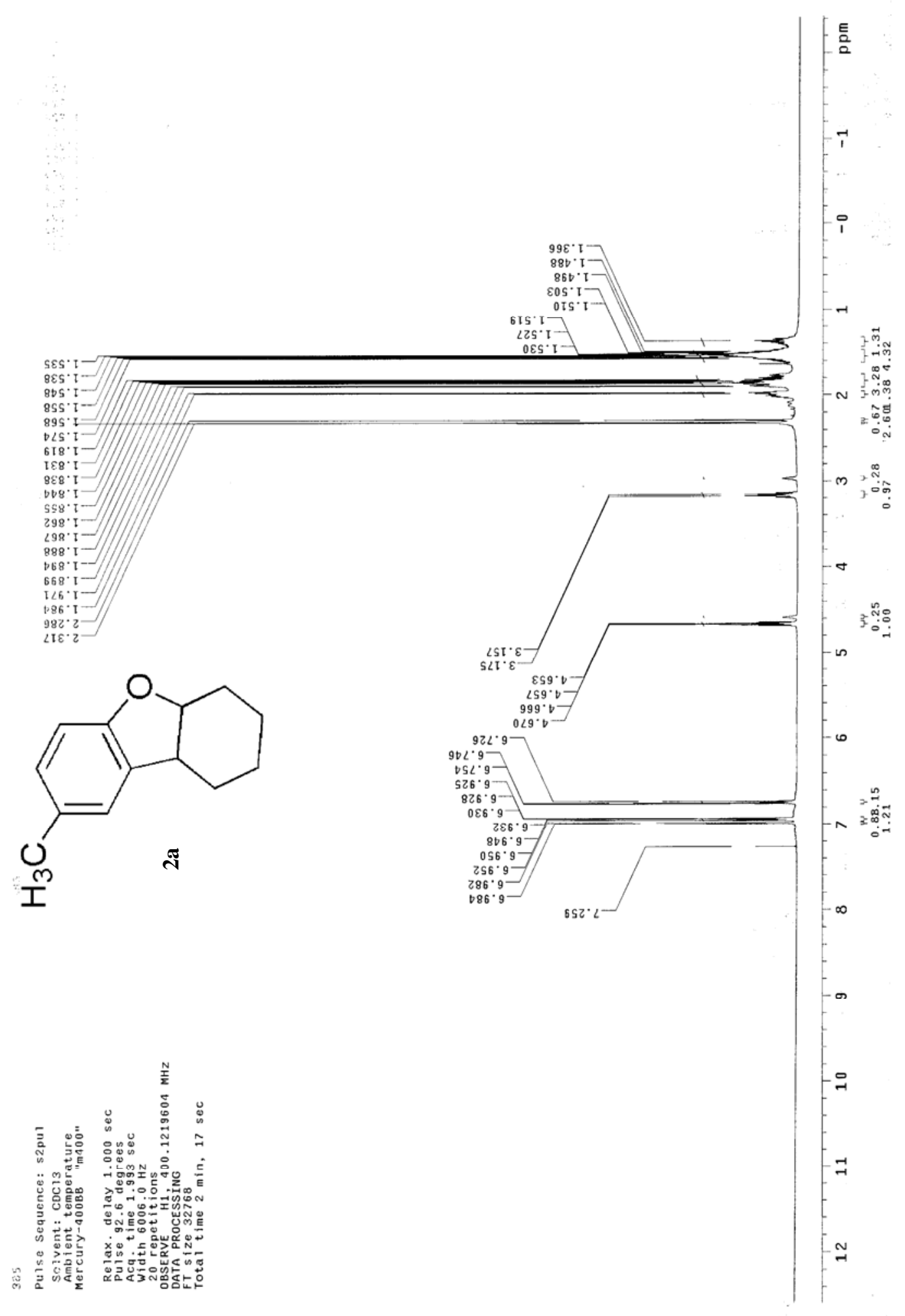




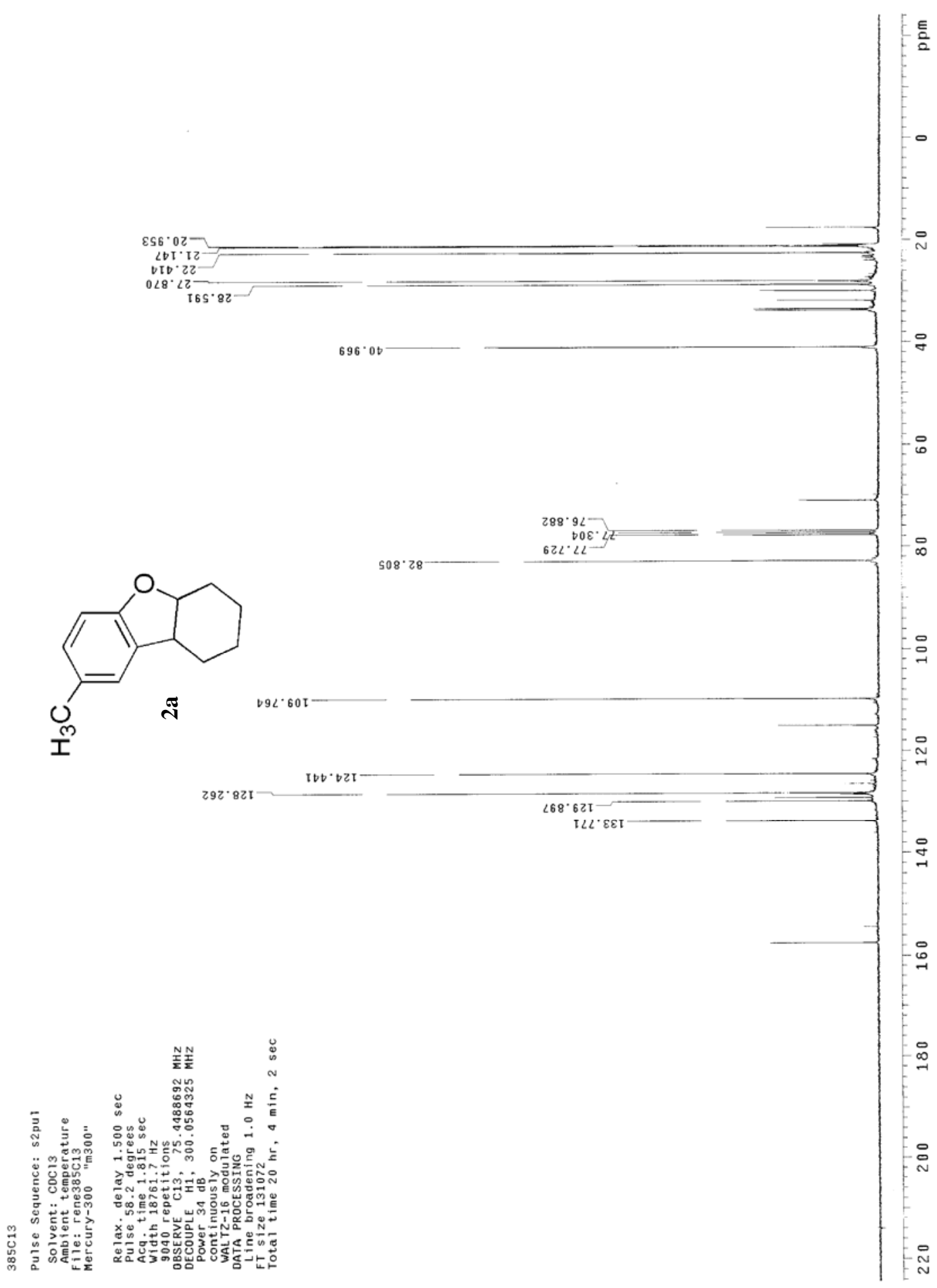




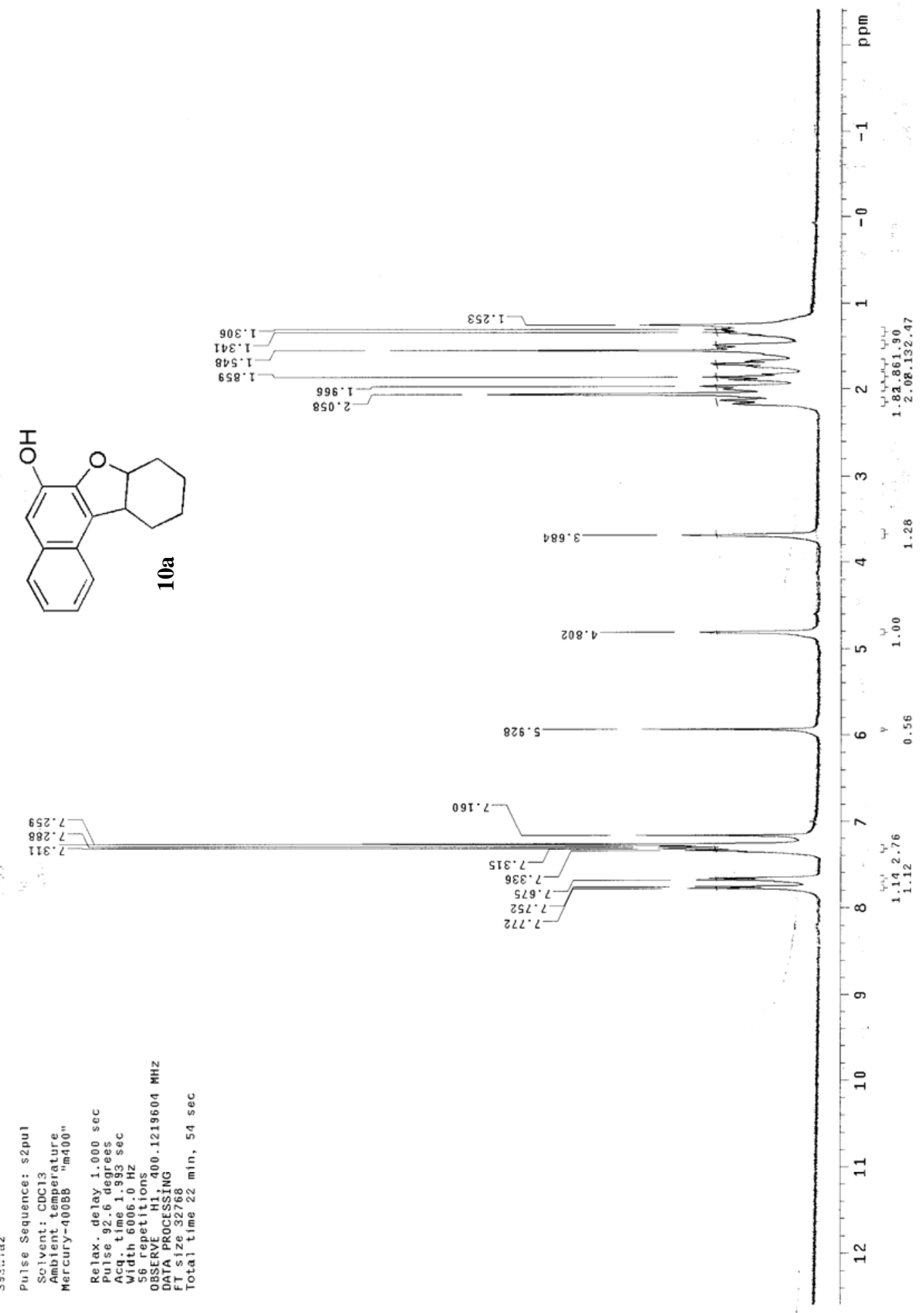




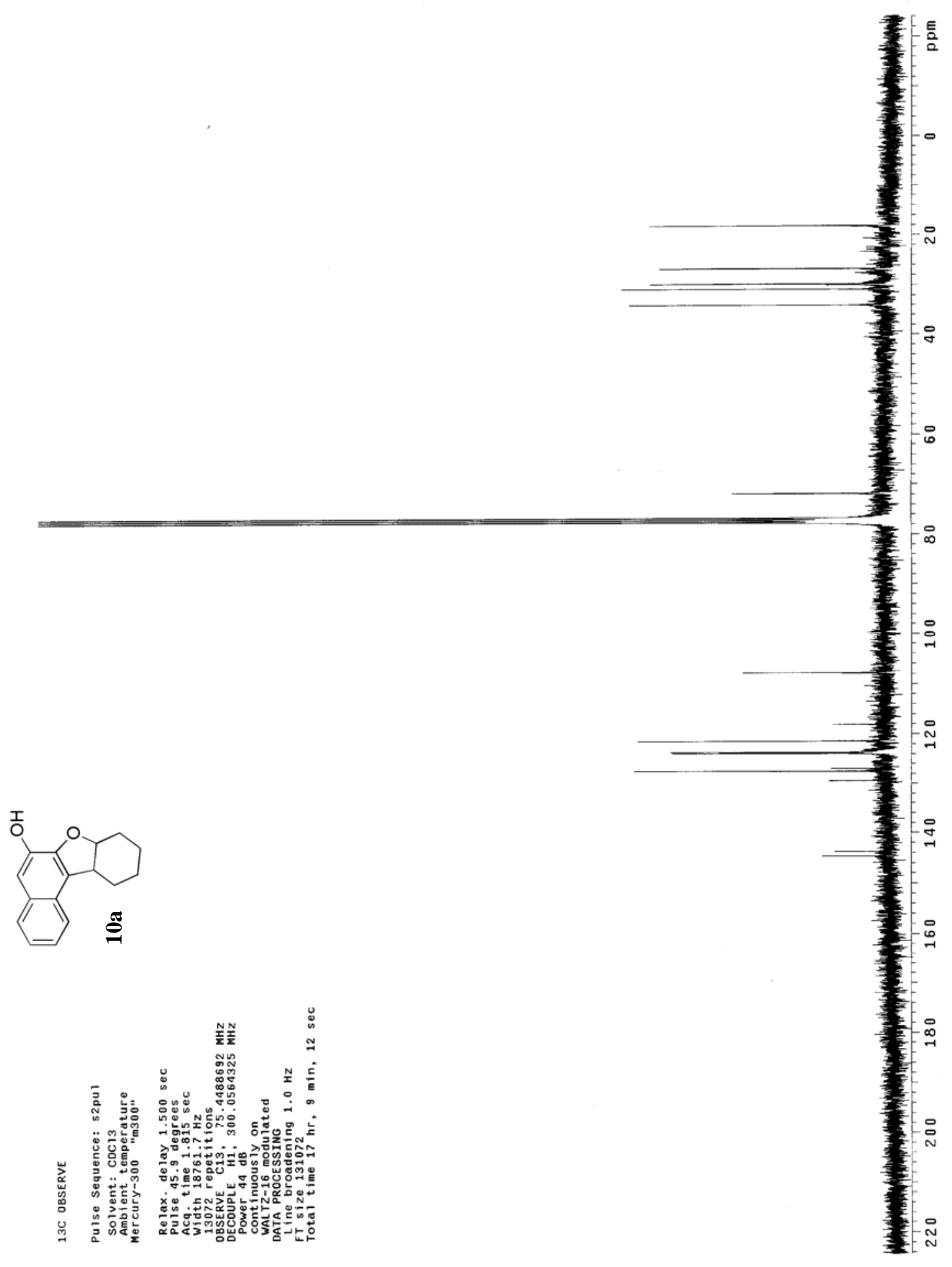




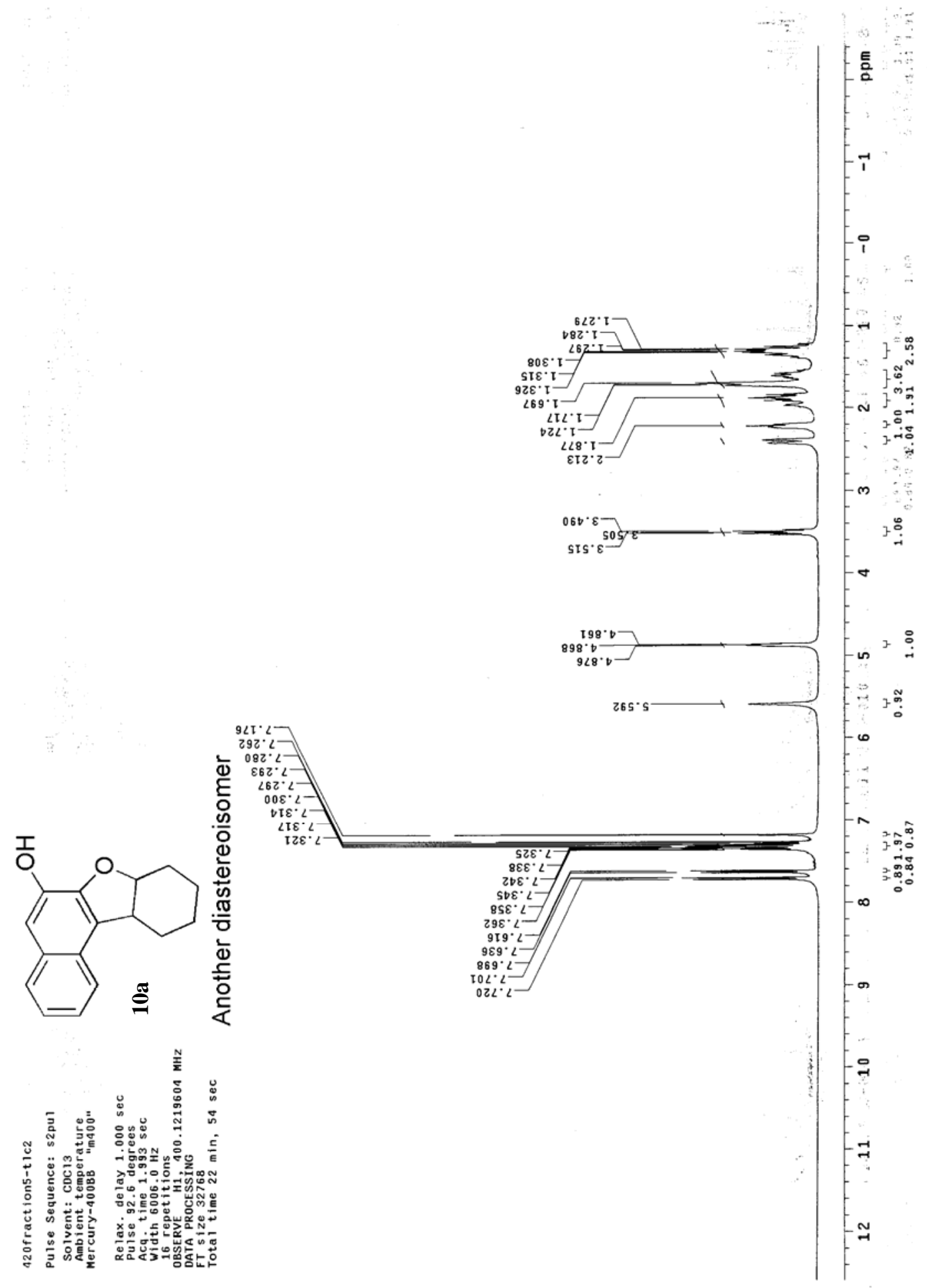




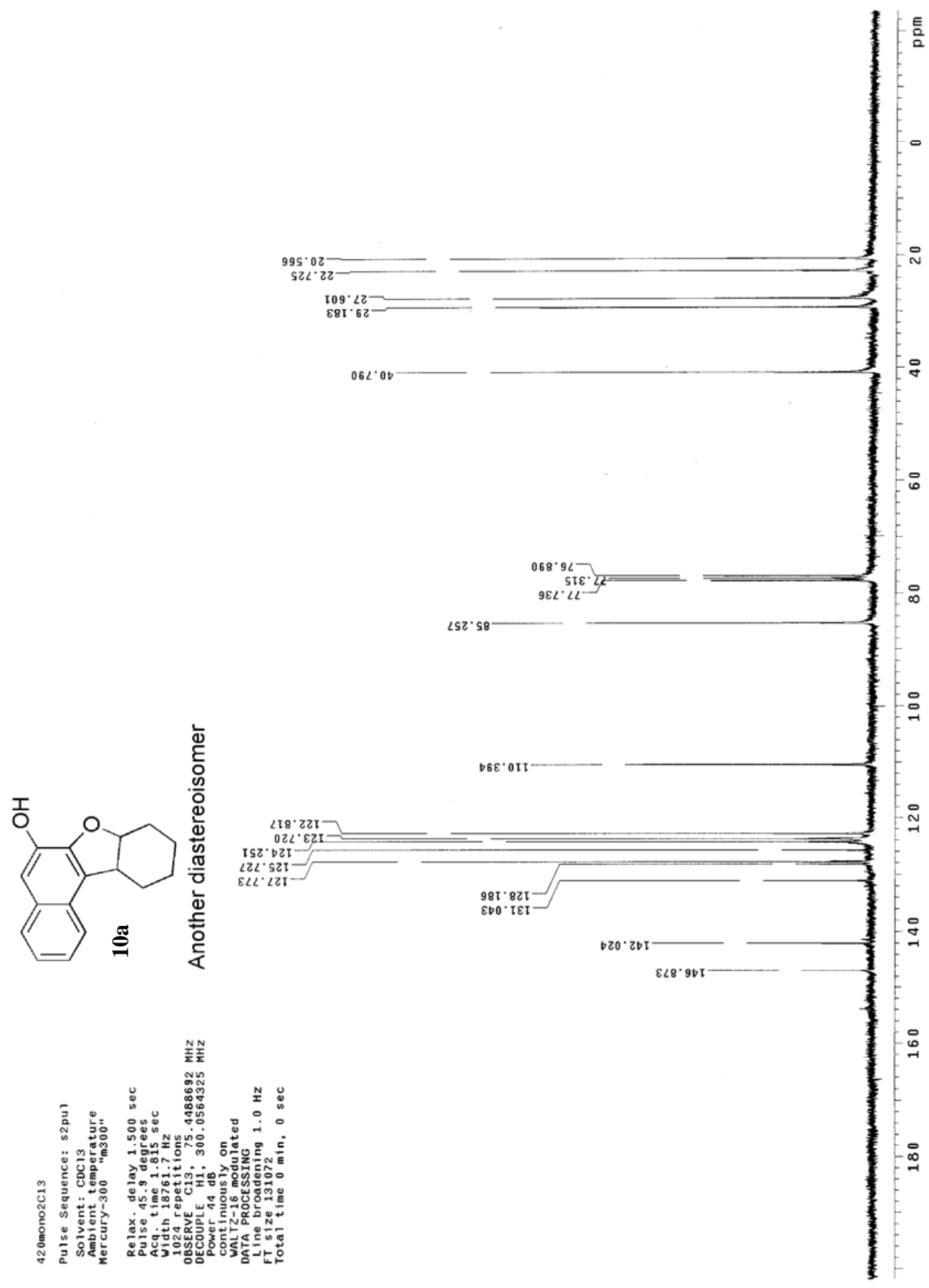




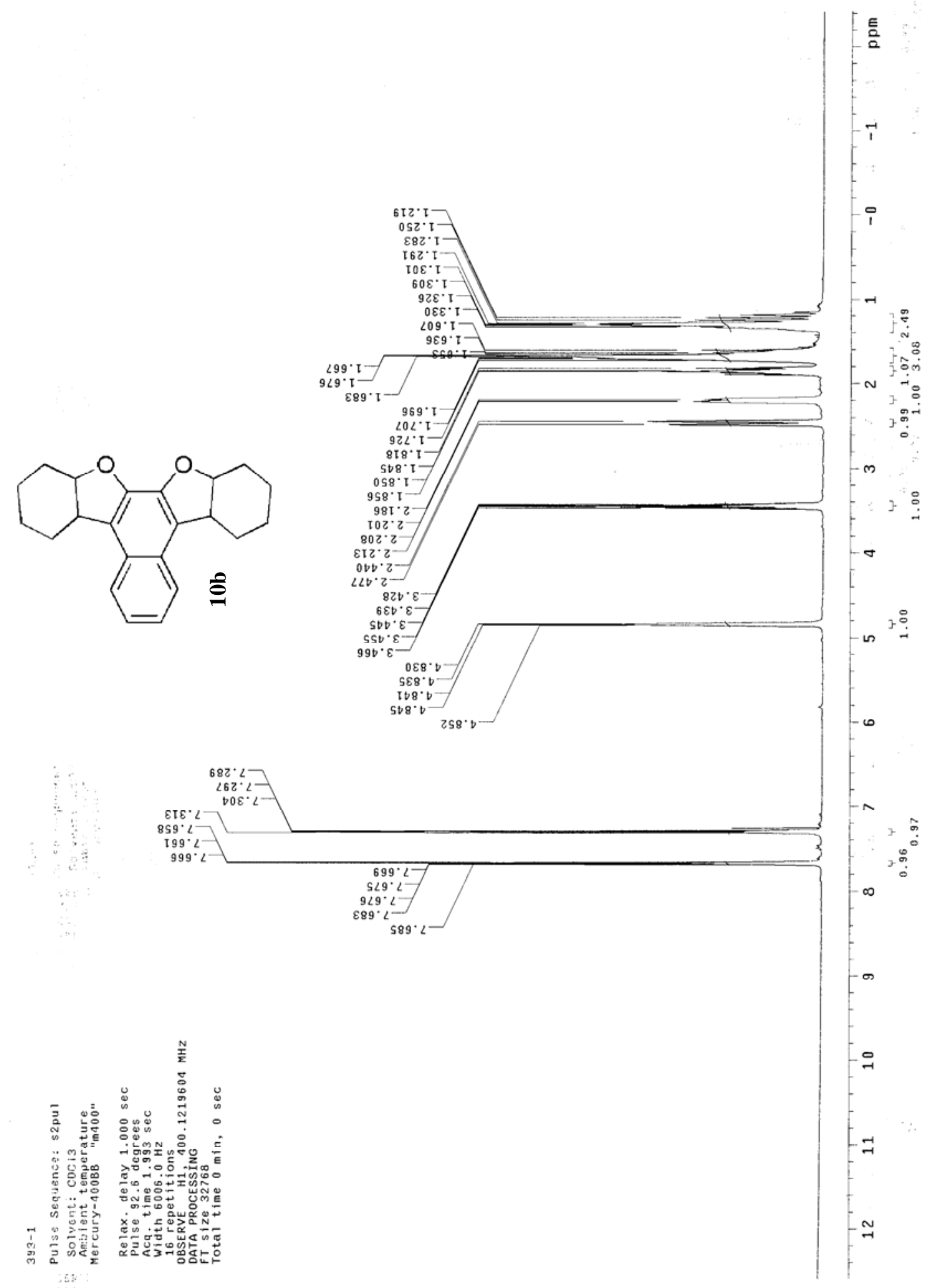




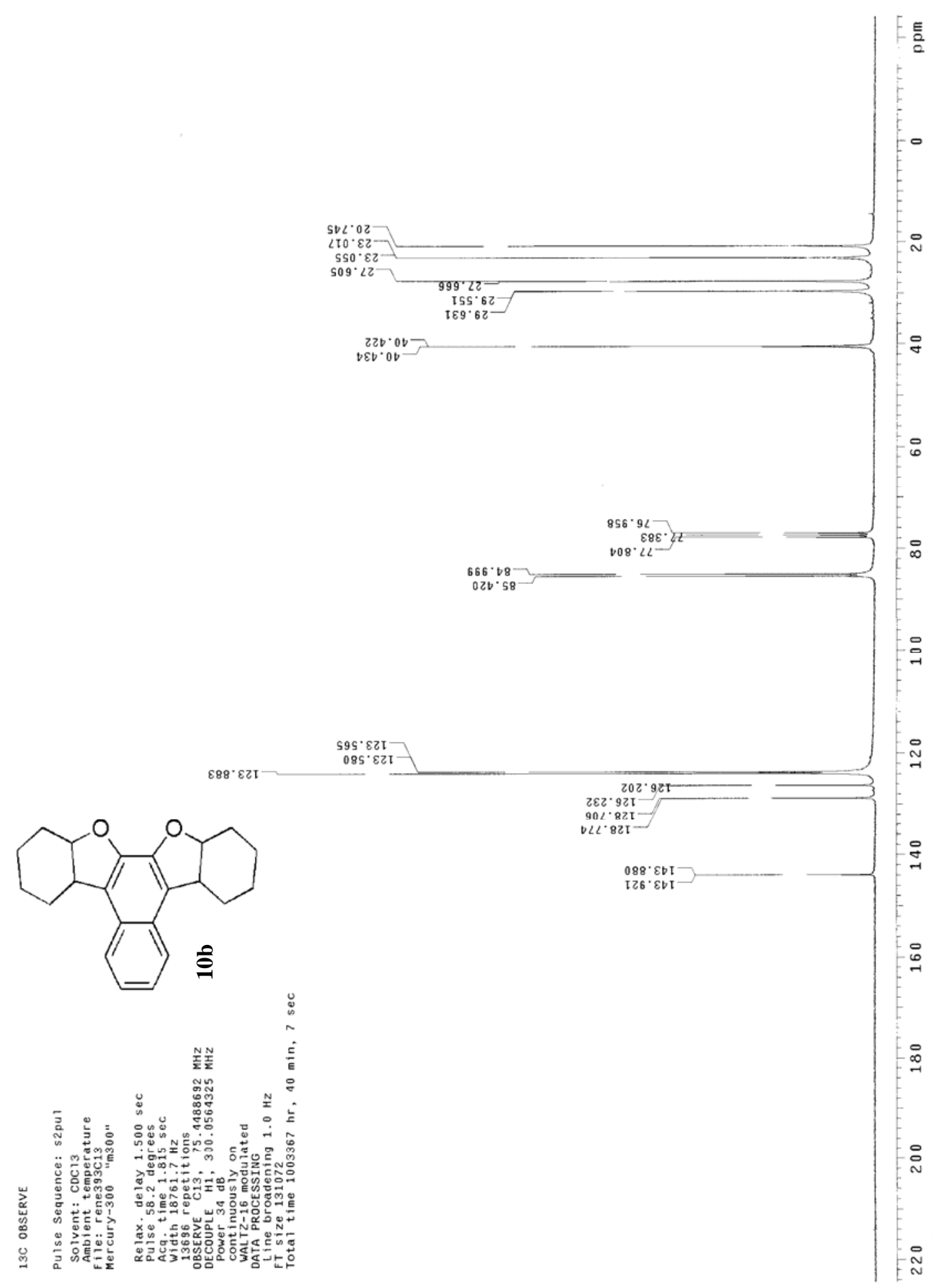




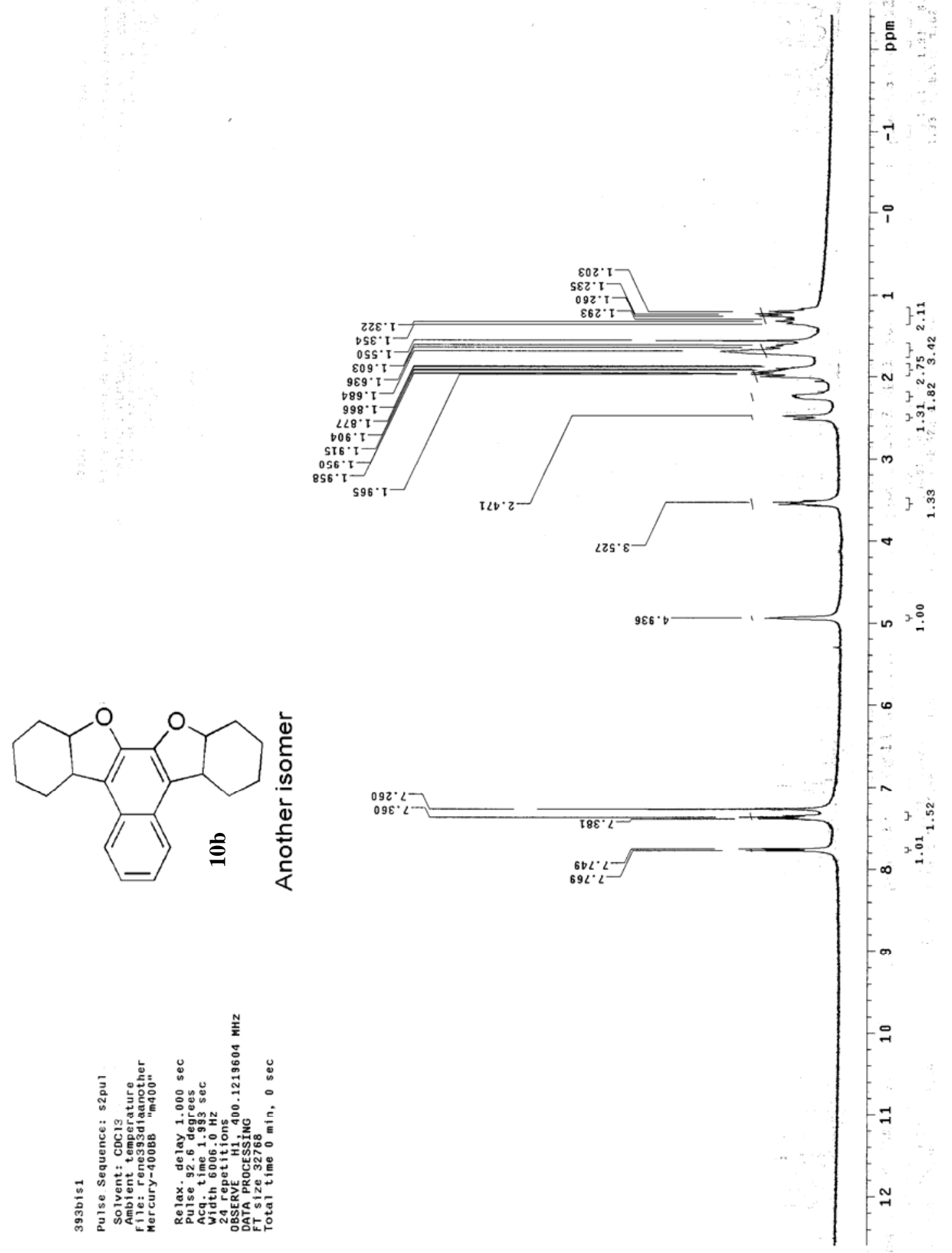




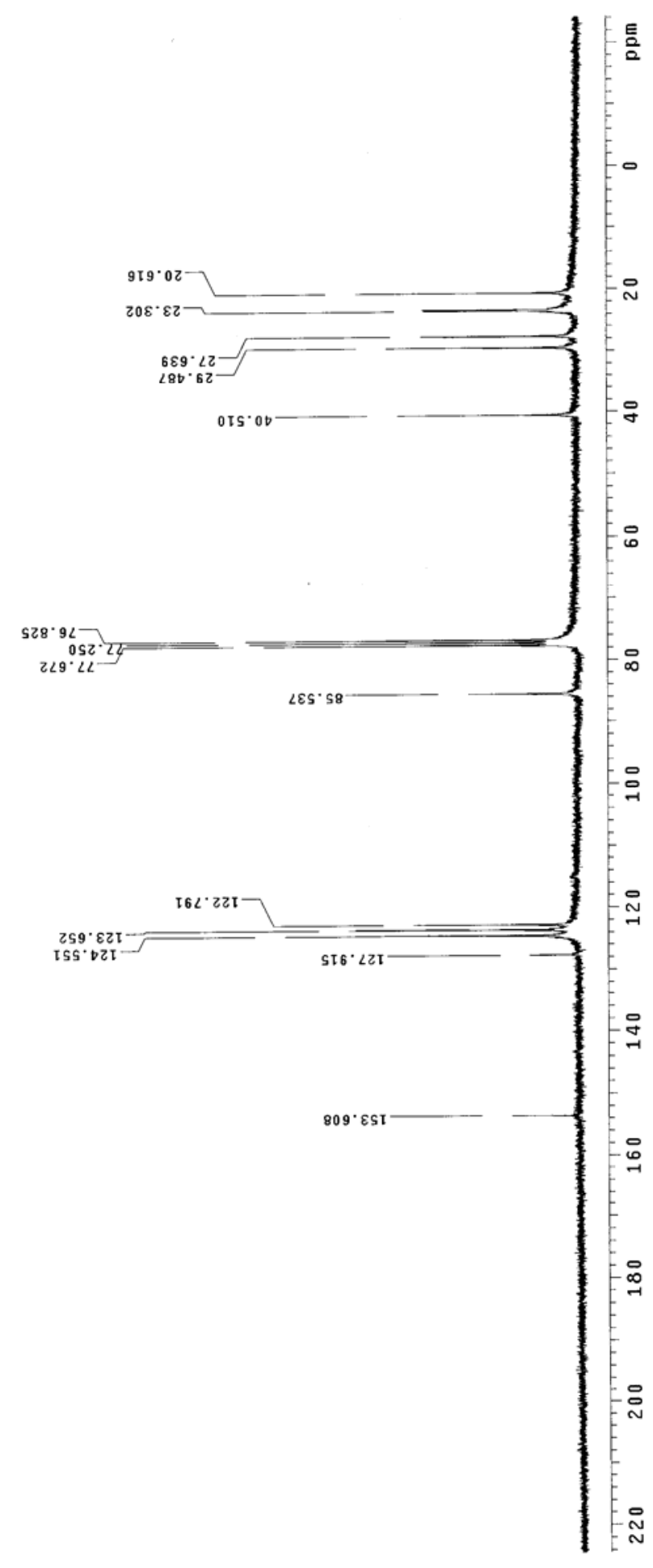




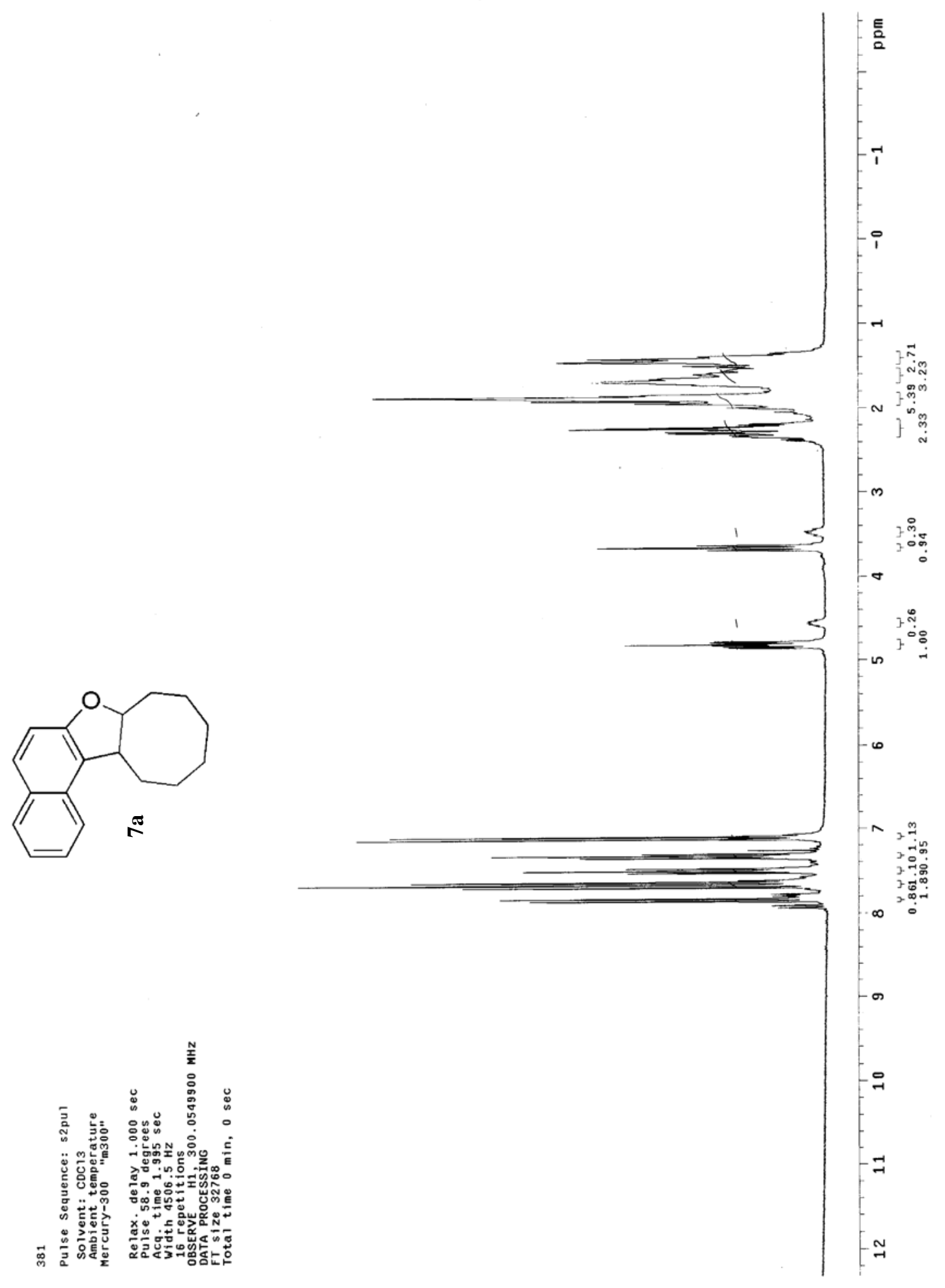




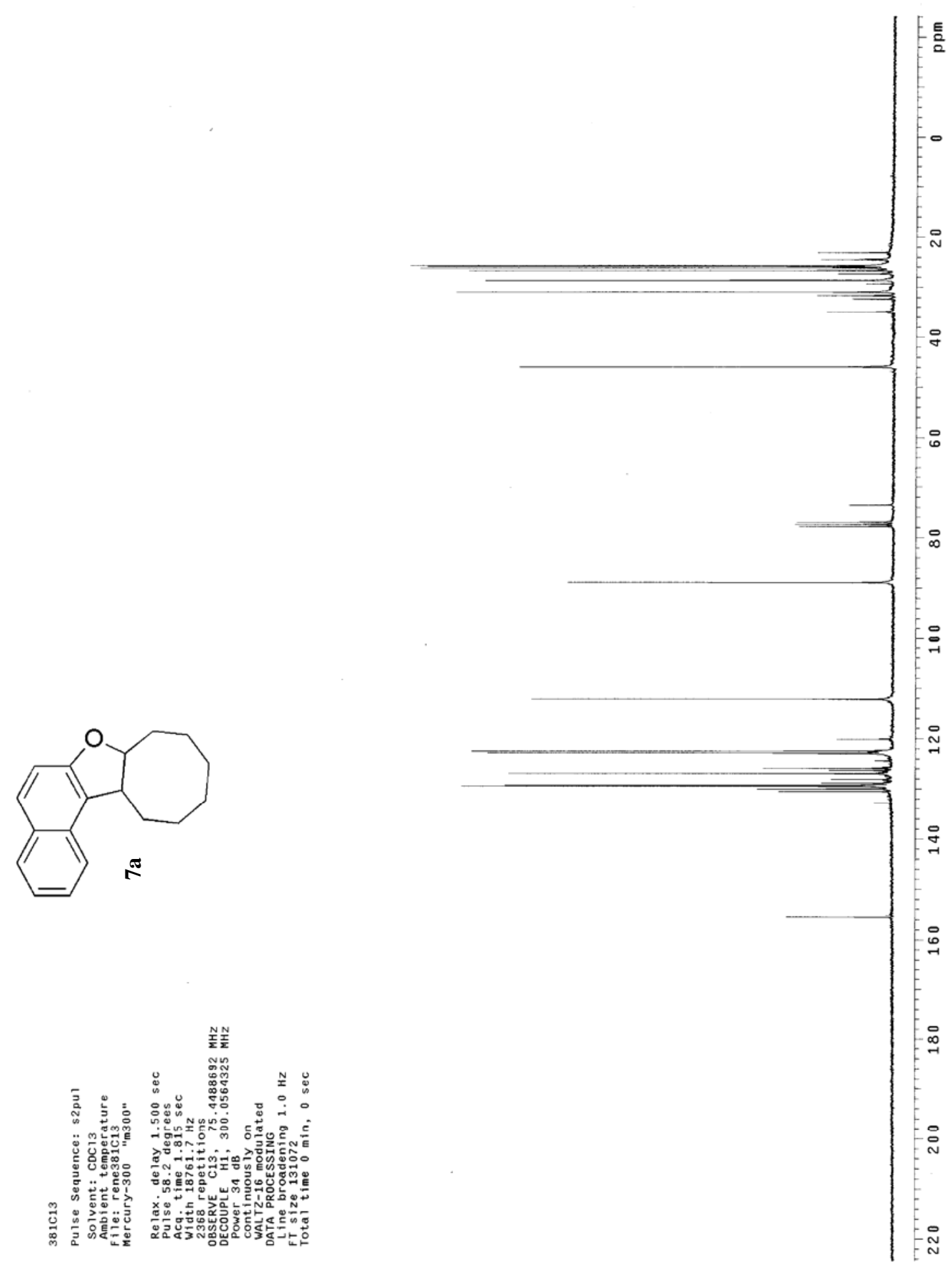




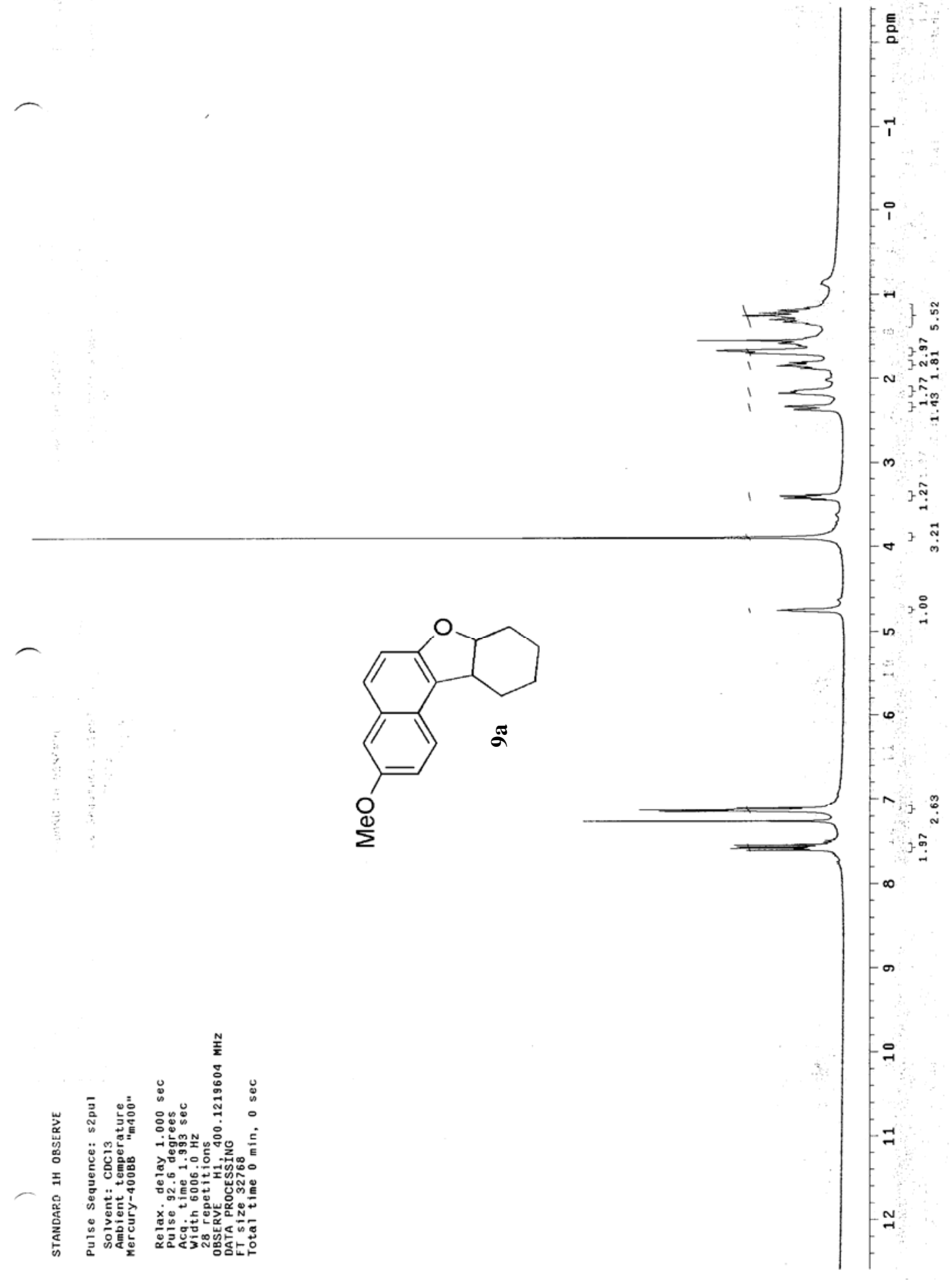




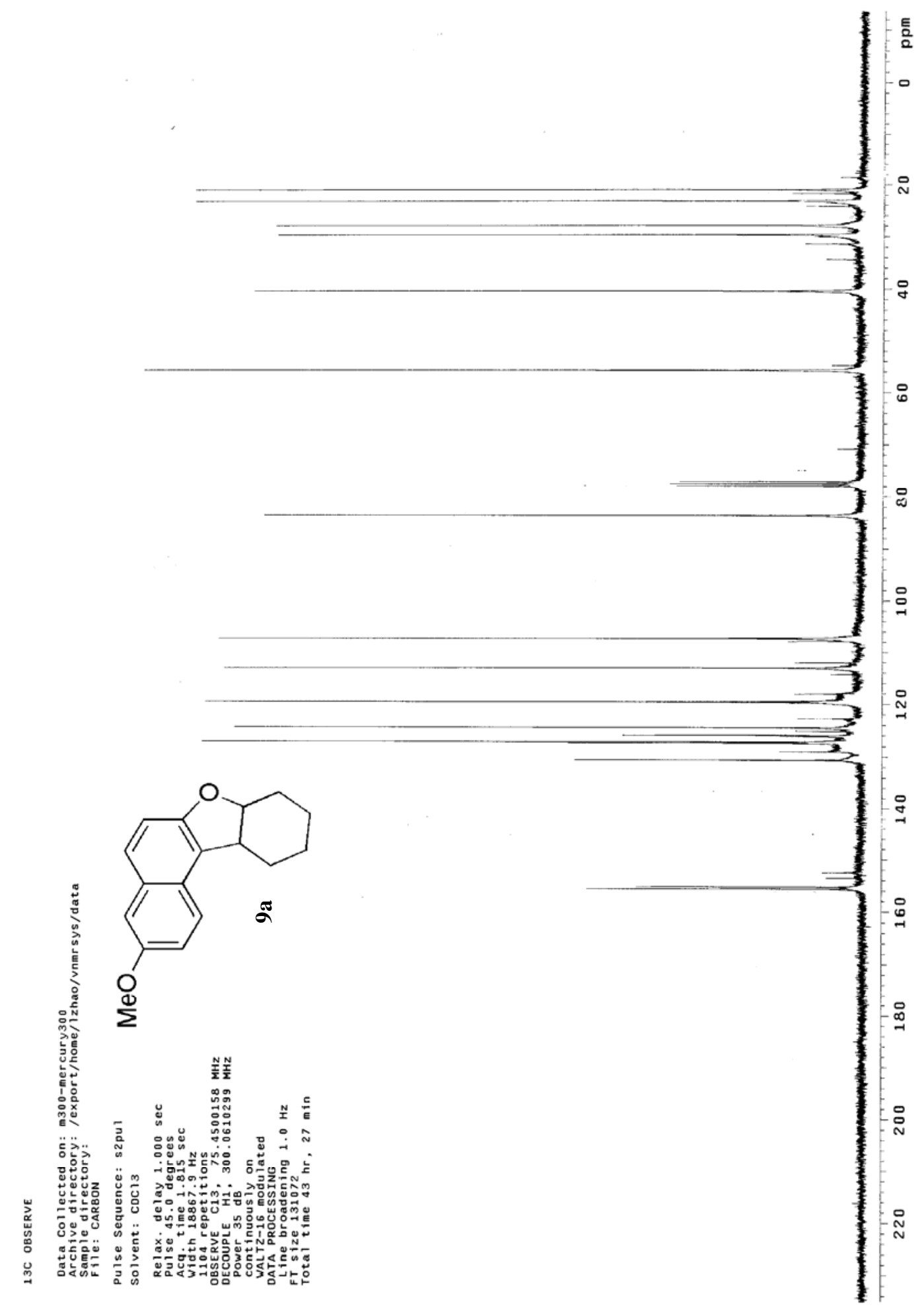

